\title{
Theory of Spatial Coherence in Near-Field Raman Scattering
}

\author{
Luiz Gustavo Cançado, ${ }^{1}$ Ryan Beams, ${ }^{2}$ Ado Jorio, ${ }^{1}$ and Lukas Novotny ${ }^{3}$ \\ ${ }^{1}$ Departamento de Física, Universidade Federal de Minas Gerais, \\ Belo Horizonte, Minas Gerais 30123-970, Brazil \\ ${ }^{2}$ Institute of Optics, University of Rochester, Rochester, New York 14627, USA \\ ${ }^{3}$ Photonics Laboratory, ETH Zürich, 8093 Zürich, Switzerland
}

(Received 28 March 2014; revised manuscript received 2 June 2014; published 26 September 2014)

\begin{abstract}
A theoretical study describing the coherence properties of near-field Raman scattering in two- and onedimensional systems is presented. The model is applied to the Raman modes of pristine graphene and graphene edges. Our analysis is based on the tip-enhanced Raman scheme, in which a sharp metal tip located near the sample surface acts as a broadband optical antenna that transfers the information contained in the spatially correlated (but nonpropagating) near field to the far field. The dependence of the scattered signal on the tip-sample separation is explored, and the theory predicts that the signal enhancement depends on the particular symmetry of a vibrational mode. The model can be applied to extract the correlation length $L_{\mathrm{c}}$ of optical phonons from experimentally recorded near-field Raman measurements. The coherence properties of optical phonons have been broadly explored in the time and frequency domains, and the spatially resolved approach presented here provides a complementary methodology for the study of local material properties at the nanoscale.
\end{abstract}

DOI: 10.1103/PhysRevX.4.031054

Raman scattering in crystals is usually treated in the literature as a spatially incoherent process $[1,2]$. In other words, the scattered field from different sample points is considered to be spatially uncorrelated. This approach is supported by the early theory of coherence stating that the field emitted by an incoherent source at a given wavelength $\lambda$ is spatially uncorrelated on length scales larger than $\lambda / 2$ (measured from the surface of the scatterer) [3]. As a consequence, correlations on length scales smaller than $\lambda / 2$ are inaccessible in standard light scattering, and the signal recorded in the far field is incoherent. With the advent of near-field optics and nanoscience in general, studies of thermal emitters revealed correlation lengths much shorter than $\lambda$ [4-8]. Here, we show that similar effects underlie near-field Raman scattering and that correlation lengths much smaller than $\lambda / 2$ can be extracted from measured data. Thus, near-field Raman scattering must take into account subwavelength correlations and associated interference effects.

The coherence of lattice vibrations is of particular importance for graphene-based electronics since the scattering of optical phonons provides the main channel for relaxation of charge carriers and heat dissipation in this material system [9-14]. In this work, we derive a theory for

Published by the American Physical Society under the terms of the Creative Commons Attribution 3.0 License. Further distribution of this work must maintain attribution to the author(s) and the published article's title, journal citation, and DOI.
Subject Areas: Condensed Matter Physics, Graphene, Optics

near-field Raman scattering in one- and two-dimensional systems and apply the theory to pristine graphene and graphene edges. Our analysis is based on the tip-enhanced Raman scattering (TERS) scheme, in which a sharp metal tip is located near the sample at distances much smaller than $\lambda$. The tip acts as a broadband optical antenna, transferring the information contained in the spatially correlated (but non propagating) near field to the far field. We analyze the dependence of the scattered signal on the tip-sample separation distance and show that different vibrational modes (with distinct symmetries and dimensionalities) give rise to different tip-sample distance dependencies. The theory has been used to measure the correlation length $L_{\mathrm{c}}$ of optical phonons in graphene, for which we found $L_{\mathrm{c}} \approx 30 \mathrm{~nm}$ [15]. Although the correlation properties of optical phonons have been broadly explored in the time and frequency domains [16], the spatially resolved approach presented here provides an alternative probe for the study of local material properties at the nanoscale.

Raman scattering is an inelastic scattering process where the incident and scattered photons present different energies. The energy difference is equal to the energy of a quantum of vibration (phonon) that is either created (Stokes Raman component) or annihilated (antiStokes Raman component) [1,2]. The scope of the present study is to extract the correlation length of phonons in crystals by exploring the coherence properties of the inelastically scattered field in Raman processes. In order to introduce the theory and the parameters involved, we 
briefly discuss the classical theory of light scattering, keeping the focus on the spatial domain.

For a linear scattering medium irradiated by a monochromatic incident beam of frequency $\omega$, the scattered field $\mathbf{E}^{\mathrm{s}}$ of frequency $\omega_{\mathrm{s}}$ that reaches the detector at the position $\mathbf{r}_{0}$ is described by the integral equation of potential scattering of the form [17]

$\mathbf{E}^{\mathrm{s}}\left(\mathbf{r}_{0} ; \omega_{\mathrm{s}}\right)=\frac{\omega_{\mathrm{s}}^{2}}{\varepsilon_{0} c^{2}} \int_{\mathbb{D}} d^{3} \mathbf{r} \stackrel{\leftrightarrow}{\mathrm{G}}\left(\mathbf{r}_{0}, \mathbf{r} ; \omega_{\mathrm{s}}\right) \mu(\mathbf{r} ; \omega) \mathbf{E}(\mathbf{r} ; \omega)$,

where $\varepsilon_{0}$ and $c$ are the free-space permittivity and speed of light, respectively, $\mu(\mathbf{r} ; \omega)$ is the scattering potential at a position $\mathbf{r}$ in the scattering domain $\mathbb{D}, \mathbf{E}(\mathbf{r} ; \omega)$ is the total exciting field at $\mathbf{r}$, and $\left.\stackrel{\mathrm{G}}{\mathbf{r}_{0}}, \mathbf{r} ; \omega_{\mathrm{s}}\right)$ is the outgoing Green function that accounts for the whole system, including the scattering and surrounding media. In the case of vibrational Raman scattering, the scattering potential is described by the second-rank polarizability tensor $\stackrel{\leftrightarrow}{\alpha}^{\gamma}$, whose components are defined as

$$
\alpha_{i j}^{\gamma}\left(\mathbf{r} ; \omega_{\mathrm{s}}, \omega\right)=\sum_{k=x, y, z} \frac{\partial \alpha_{i j}(\mathbf{r} ; \omega)}{\partial q_{k}} q_{k},
$$

with $\alpha$ being the polarizability per unit area at frequency $\omega$ and $\mathbf{q}=\left(q_{x}, q_{y}, q_{z}\right)$ being the lattice displacement vector associated with a particular vibrational mode $\gamma$ with frequency $\left|\omega-\omega_{\mathrm{s}}\right|$. Notice that the product $\stackrel{\leftrightarrow}{\alpha} \gamma\left(\mathbf{r} ; \omega_{\mathrm{s}}, \omega\right) \times$ $\mathbf{E}(\mathbf{r} ; \omega)$ defines the induced Raman dipole per unit area $\mathbf{p}^{\gamma}\left(\mathbf{r} ; \omega_{\mathrm{s}}, \omega\right)$, and in this case, Eq. (1) assumes the form

$$
\mathbf{E}^{\mathrm{s}}\left(\mathbf{r}_{0} ; \omega_{\mathrm{s}}\right)=\frac{\omega_{\mathrm{s}}^{2}}{\varepsilon_{0} c^{2}} \int_{\mathbb{D}} d^{3} \mathbf{r} \stackrel{\leftrightarrow}{\mathrm{G}}\left(\mathbf{r}_{0}, \mathbf{r} ; \omega_{\mathrm{s}}\right) \mathbf{p}^{\gamma}\left(\mathbf{r} ; \omega_{\mathrm{s}}, \omega\right),
$$

with

$$
\mathbf{p}^{\gamma}\left(\mathbf{r} ; \omega_{\mathrm{s}}, \omega\right)=\stackrel{\leftrightarrow}{\alpha}^{\gamma}\left(\mathbf{r} ; \omega_{\mathrm{s}}, \omega\right) \mathbf{E}(\mathbf{r} ; \omega)
$$

By considering the field as a single realization in the spectral domain, the detector renders a signal $S\left(\mathbf{r}_{0} ; \omega_{\mathrm{s}}\right)$ that is proportional to the ensemble average of the scattered field (3):

$$
\begin{aligned}
S\left(\mathbf{r}_{0} ; \omega_{\mathrm{s}}\right)= & \left\langle\mathbf{E}^{\mathrm{s} *}\left(\mathbf{r}_{0}, \omega_{\mathrm{s}}\right) \cdot \mathbf{E}^{\mathrm{s}}\left(\mathbf{r}_{0}, \omega_{\mathrm{s}}\right)\right\rangle \\
= & \frac{\omega_{\mathrm{s}}^{2}}{\varepsilon_{0} c^{2}} \int_{\mathbb{D}} d^{3} \mathbf{r}_{1} \int_{\mathbb{D}} d^{3} \mathbf{r}_{2}\left\langle\stackrel{\leftrightarrow}{\mathrm{G}}\left(\mathbf{r}_{0}, \mathbf{r}_{1} ; \omega_{\mathrm{s}}\right)\right. \\
& \left.\times \mathbf{p}^{\gamma}\left(\mathbf{r}_{1} ; \omega_{\mathrm{s}}, \omega\right) \cdot \stackrel{\leftrightarrow}{\mathrm{G}}\left(\mathbf{r}_{0}, \mathbf{r}_{2} ; \omega_{\mathrm{s}}\right) \mathbf{p}^{\gamma}\left(\mathbf{r}_{2} ; \omega_{\mathrm{s}}, \omega\right)\right\rangle
\end{aligned}
$$

Figure 1 illustrates two individual scattering events, where the scattering domain $\mathbb{D}$ is irradiated by the field $\mathbf{E}(\omega)$. On length scales $\left|\mathbf{r}_{1}-\mathbf{r}_{2}\right|$ smaller than the phonon correlation

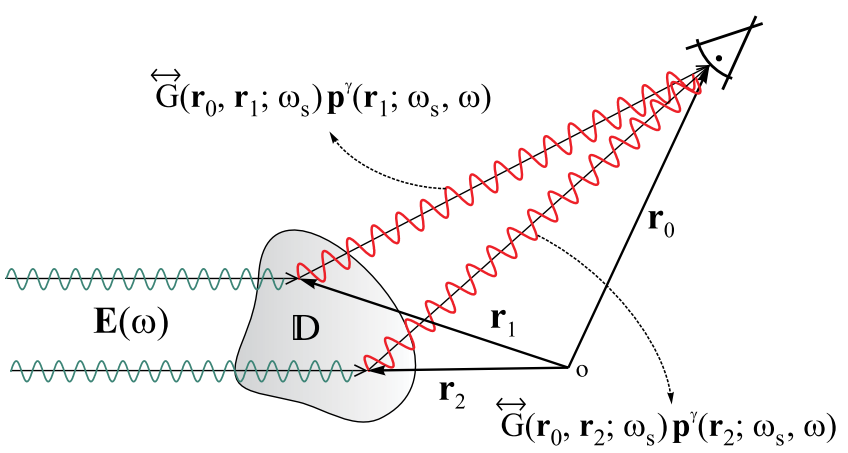

FIG. 1. Illustration of two individual scattering paths associated with a scatterer $\mathbb{D}$ irradiated by the field $\mathbf{E}(\omega)$. On length scales $\left|\mathbf{r}_{1}-\mathbf{r}_{2}\right|$ smaller than the phonon correlation length $L_{\mathrm{c}}$ of a vibrational mode $\gamma$, the partial fields $\stackrel{\leftrightarrow}{\mathrm{G}}\left(\mathbf{r}_{0}, \mathbf{r}_{1} ; \omega_{\mathrm{s}}\right) \mathbf{p}^{\gamma}\left(\mathbf{r}_{1} ; \omega_{\mathrm{s}}, \omega\right)$ and $\stackrel{\leftrightarrow}{\mathrm{G}}\left(\mathbf{r}_{0}, \mathbf{r}_{2} ; \omega_{\mathrm{s}}\right) \mathbf{p}^{\gamma}\left(\mathbf{r}_{2} ; \omega_{\mathrm{s}}, \omega\right)$ add coherently at location $\mathbf{r}_{0}$ of the detector. On the other hand, for length scales larger than $L_{\mathrm{c}}$, there is no phase correlation between the scattering events; hence, the partial fields at the detector add incoherently.

length $L_{\mathrm{c}}$, the partial fields $\stackrel{\leftrightarrow}{\mathrm{G}}\left(\mathbf{r}_{0}, \mathbf{r}_{1} ; \omega_{\mathrm{s}}\right) \mathbf{p}^{\gamma}\left(\mathbf{r}_{1} ; \omega_{\mathrm{s}}, \omega\right)$ and $\stackrel{\leftrightarrow}{\mathrm{G}}\left(\mathbf{r}_{0}, \mathbf{r}_{2} ; \omega_{\mathrm{s}}\right) \mathbf{p}^{\gamma}\left(\mathbf{r}_{2} ; \omega_{\mathrm{s}}, \omega\right)$ add coherently at the detector. On the other hand, for length scales larger than $L_{\mathrm{c}}$, there is no phase correlation between the dipoles, and hence, the partial fields at the detector add incoherently. For experiments using a coherent exciting field (laser source), the signal becomes [Eqs. (4) and (5)]

$$
\begin{aligned}
S\left(\mathbf{r}_{0}, \omega_{\mathrm{s}}\right)= & \frac{\omega_{\mathrm{s}}^{4}}{\varepsilon_{0}^{2} c^{4}} \sum_{l, m, n} \sum_{i, j} \int_{\mathbb{D}} d^{3} \mathbf{r}_{2} \int_{\mathbb{D}} d^{3} \mathbf{r}_{1}\left\langle\alpha_{m i}^{\gamma *}\left(\mathbf{r}_{1}, \omega_{\mathrm{s}}\right)\right. \\
& \left.\times \alpha_{n j}^{\gamma}\left(\mathbf{r}_{2}, \omega_{\mathrm{s}}\right)\right\rangle \mathrm{G}_{l m}^{*}\left(\mathbf{r}_{0}, \mathbf{r}_{1} ; \omega_{\mathrm{s}}\right) \mathrm{G}_{l n}\left(\mathbf{r}_{0}, \mathbf{r}_{2} ; \omega_{\mathrm{s}}\right) \\
& \times E_{i}^{*}\left(\mathbf{r}_{1}, \omega\right) E_{j}\left(\mathbf{r}_{2}, \omega\right),
\end{aligned}
$$

with $l, m, n \in\{x, y, z\}$. Equation (6) tells us that, for experiments carried out with an incident laser beam, the spatial coherence of the scattered signal is solely described by the correlation of the Raman polarizability tensor components.

Classical textbooks describing Raman scattering usually do not consider the spatial coherence of the scattered field (see, for example, Refs. [1,2]). The correlation function $\left\langle\alpha_{m i}^{\gamma *} \alpha_{n j}^{\gamma}\right\rangle$ is typically assumed to be a Dirac delta function, for which the signal in Eq. (6) turns into a simple integration over the scattering volume $V_{\mathbb{D}}$. The outgoing Green function accounts for the polarization direction of the scattered field (defined by the unit vector $\hat{\mathbf{\epsilon}}_{\mathrm{s}}$ ) in the presence of analyzers, and also for the solid angle $\Omega$ of the collection optics. Equation (6) is then reduced to $[1,2]$

$$
S\left(\mathbf{r}_{0}, \omega_{\mathrm{s}}\right) \propto V_{\mathbb{D}} \Omega \frac{\omega_{\mathrm{s}}^{4}}{\varepsilon_{0}^{2} c^{4}}\left|\hat{\mathbf{\epsilon}}_{\mathrm{s}} \cdot \stackrel{\leftrightarrow}{\alpha}\left(\omega_{\mathrm{s}}, \omega\right) \mathbf{E}(\omega)\right|^{2}
$$


Indeed, spatial correlations associated with vibrational states can be neglected in usual Raman scattering experiments performed in the far-field regime, since the correlation length $L_{\mathrm{c}}$ of optical phonons in crystals is on the order of tens of nanometers, one order of magnitude shorter than the wavelength of visible light. In the case of Raman scattering of liquids and gases, this approximation is even better applied since the correlation length associated with vibrational states of the molecules contained in these systems is in the range of a few nanometers, defined by thermal fluctuations.

The most important point here is that the analyses performed in Refs. [1,2] do not take into account the nonradiating near-field components in the light-matter interaction, and therefore, Eq. (7) hides important information related to the spatial correlation on length scales smaller than $\lambda / 2$. To account for spatial coherence in near-field Raman scattering, we will consider Gaussian correlations of the form

$$
\begin{aligned}
\left\langle\alpha_{m i}^{\gamma *}\left(\mathbf{r}_{1}, \omega_{\mathrm{s}}\right) \alpha_{n j}^{\gamma}\left(\mathbf{r}_{2}, \omega_{\mathrm{s}}\right)\right\rangle= & \tilde{\alpha}_{m i}^{\gamma *}\left(\mathbf{r}_{1}, \omega_{\mathrm{s}}\right) \tilde{\alpha}_{n j}^{\gamma}\left(\mathbf{r}_{2}, \omega_{\mathrm{s}}\right) \\
& \times \frac{\mathrm{e}^{-\left(\left|\mathbf{r}_{1}-\mathbf{r}_{2}\right|^{2} / L_{\mathrm{c}}^{2}\right)}}{\pi L_{\mathrm{c}}^{2}} .
\end{aligned}
$$

The last term turns into a spatial delta function in the limit $L_{\mathrm{c}} \rightarrow 0$ and into a constant term for $L_{\mathrm{c}} \rightarrow \infty$.

In the following, we will consider monolayer graphene as our material system. The well-defined Raman modes of graphene provide an excellent model system for our theory since they involve distinct symmetries and dimensionalities. The three main features present in the Raman spectrum of graphene are $[18,19]$ (i) the one-phonon first-order allowed $\mathrm{G}$ band (around $1580 \mathrm{~cm}^{-1}$ ) originating from the double-degenerate bond-stretching mode with $\mathrm{E}_{2 \mathrm{~g}}$ symmetry, occurring at the center of the Brillouin zone ( $\Gamma$ point) where the transverse optical (TO) and longitudinal optical (LO) phonon branches touch each other, (ii) the disorder-induced $\mathrm{D}$ band (about $1350 \mathrm{~cm}^{-1}$ ) originating from totally symmetric $\left(\mathrm{A}_{1}\right)$ TO phonons occurring near the edges ( $\mathrm{K}$ and $\mathrm{K}^{\prime}$ points) of the first Brillouin zone, activated by structural defects that provide momentum conservation in a double-resonance process, and (iii) the two-phonon $\mathrm{G}^{\prime}$ band (also called 2D in the literature, but not here where 2D stands for two-dimensional) centered at about $2700 \mathrm{~cm}^{-1}$, generated by triple-resonance processes in monolayer graphene and related to the same phonon as the D band,

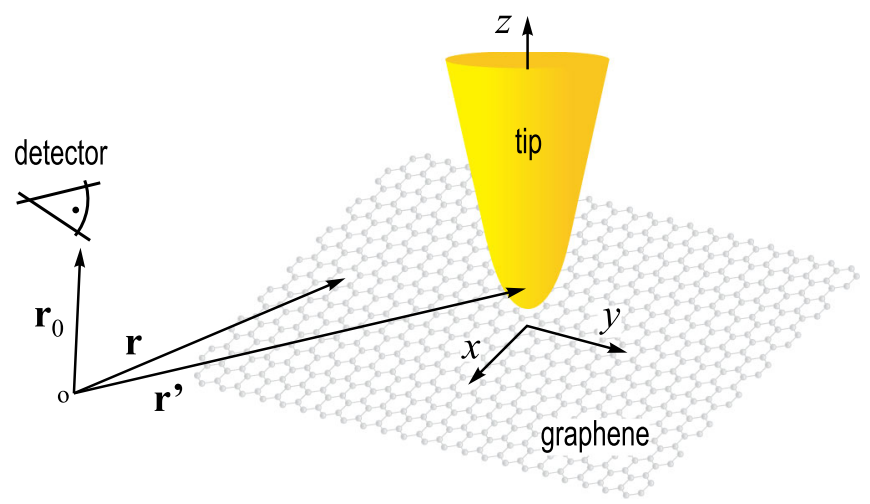

FIG. 2. Illustration of spatially resolved near-field Raman scattering of a graphene sample. The electric field $\mathbf{E}$ confined to the apex of a laser-irradiated gold tip interacts locally with the graphene lattice characterized by the Raman polarizability $\stackrel{\leftrightarrow}{\alpha}^{\gamma}$, where $\gamma$ denotes a specific phonon mode $\left(\gamma \in\left[\mathrm{G}, \mathrm{D}, \mathrm{G}^{\prime}\right]\right)$.

although it does not require the presence of defects for its activation. While the $\mathrm{G}$ and $\mathrm{G}^{\prime}$ bands are allowed over the entire graphene lattice, the defect-induced D band is strongly localized near the graphene edges, which gives it a one-dimensional character [20-23]. For this reason, we refer to $G$ and $G^{\prime}$ as two dimensional (2D), whereas $D$ is denoted as a one-dimensional (1D) mode. The corresponding Raman polarizability tensors associated with these Raman modes can be represented as [24]

$$
\begin{aligned}
& \stackrel{\leftrightarrow}{\alpha}\left(\mathrm{E}_{2 \mathrm{~g} 1}\right)=\alpha^{\mathrm{G}}\left[\begin{array}{rr}
1 & 0 \\
0 & -1
\end{array}\right], \quad \stackrel{\leftrightarrow}{\alpha}\left(\mathrm{E}_{2 \mathrm{~g} 2}\right)=\alpha^{\mathrm{G}}\left[\begin{array}{ll}
0 & 1 \\
1 & 0
\end{array}\right], \\
& \stackrel{\leftrightarrow}{\alpha}^{\mathrm{D}, \mathrm{G}^{\prime}}\left(\mathrm{A}_{1}\right)=\alpha^{\mathrm{D}, \mathrm{G}^{\prime}}\left[\begin{array}{ll}
1 & 0 \\
0 & 1
\end{array}\right] \text {, }
\end{aligned}
$$

where $E_{2 \mathrm{~g} 1}$ and $\mathrm{E}_{2 \mathrm{~g} 2}$ are the two components of the doubledegenerate $E_{2 g}$ mode giving rise to the $G$ band. We have omitted the $i z$ and $z j$ components in Eq. (9) because they are null.

Figure 2 illustrates a near-field Raman experiment on a graphene sample where, by placing the origin of the coordinate system in the graphene plane right under the tip, $\mathbf{r}^{\prime}=(0,0, z)$ denotes the position of the near-field probe, $\mathbf{r}_{0}$ is the location of the detector, and $\mathbf{r}=(x, y, 0)$ is a point on the sample plane. The insertion of the Gaussian correlation function (8) into the expression for the signal (6) yields

$$
\begin{aligned}
S\left(\mathbf{r}_{0}, \omega_{\mathrm{s}}\right)= & \frac{\omega_{\mathrm{s}}^{4}}{\varepsilon_{0}^{2} c^{4}} \sum_{l, m, n} \sum_{i, j} \iint_{-\infty}^{+\infty} d x_{2} d y_{2} \mathrm{G}_{l n}\left(\mathbf{r}_{0}, x_{2}, y_{2} ; \omega_{\mathrm{s}}\right) \tilde{\alpha}_{n j}^{\gamma} E_{j}\left(x_{2}, y_{2}, \omega\right) \\
& \times \iint_{-\infty}^{+\infty} d x_{1} d y_{1} \frac{\mathrm{e}^{-\left[\left(x_{1}-x_{2}\right)^{2}+\left(y_{1}-y_{2}\right)^{2}\right] / L_{\mathrm{c}}^{2}}}{\pi L_{\mathrm{c}}^{2}} \mathrm{G}_{l m}^{*}\left(\mathbf{r}_{0}, x_{1}, y_{1} ; \omega_{\mathrm{s}}\right) \tilde{\alpha}_{m i}^{\gamma *} E_{i}^{*}\left(x_{1}, y_{1}, \omega\right),
\end{aligned}
$$

where $l \in\{x, y, z\}$ and $m, n ; i, j \in\{x, y\}$. 
To simplify notation, we will suppress the frequencies $\omega$ and $\omega_{\mathrm{s}}$ in the arguments of the different functions. We introduce the Fourier transform of the correlation function

$$
\frac{1}{4 \pi^{2}} \iint_{-\infty}^{+\infty} d x_{2} d y_{2}\left\langle\alpha_{m i}^{\gamma *}\left(x_{1}, y_{1}\right) \alpha_{n j}^{\gamma}\left(x_{2}, y_{2}\right)\right\rangle \mathrm{e}^{-i\left(k_{x} x_{2}+k_{y} y_{2}\right)}=\frac{\tilde{\alpha}_{m i}^{\gamma *} \tilde{\alpha}_{n j}^{\gamma}}{4 \pi^{2}} \mathrm{e}^{-i\left(k_{x} x_{1}+k_{y} y_{1}\right)-\left(k_{x}^{2}+k_{y}^{2}\right) L_{c}^{2} / 4},
$$

with $\tilde{\alpha}_{i j}^{\gamma}$ defined by the elements of the Raman polarizability tensors (9). The inverse transform of Eq. (11) is given as

$$
\left\langle\alpha_{m i}^{\gamma *}\left(x_{1}, y_{1}\right) \alpha_{n j}^{\gamma}\left(x_{2}, y_{2}\right)\right\rangle=\iint_{-\infty}^{+\infty} d k_{x} d k_{y} \frac{\tilde{\alpha}_{m i}^{\gamma *} \tilde{\alpha}_{n j}^{\gamma}}{4 \pi^{2}} \mathrm{e}^{-\left(k_{x}^{2}+k_{y}^{2}\right) L_{\mathrm{c}}^{2} / 4} \mathrm{e}^{i\left(k_{x}\left[x_{2}-x_{1}\right]+k_{y}\left[y_{2}-y_{1}\right]\right)} .
$$

Likewise, we introduce the Fourier transform

$$
\hat{F}_{l n j}\left(k_{x}, k_{y}\right)=\frac{1}{4 \pi^{2}} \iint_{-\infty}^{+\infty} d x_{2} d y_{2} \mathrm{G}_{l n}\left(\mathbf{r}_{0}, x_{2}, y_{2}\right) E_{j}\left(x_{2}, y_{2}\right) \mathrm{e}^{-i\left(k_{x} x_{2}+k_{y} y_{2}\right)},
$$

with the corresponding inverse transform

$$
\mathrm{G}_{l n}\left(\mathbf{r}_{0}, x_{2}, y_{2}\right) E_{j}\left(x_{2}, y_{2}\right)=\iint_{-\infty}^{+\infty} d k_{x} d k_{y} \hat{F}_{l n j}\left(k_{x}, k_{y}\right) \mathrm{e}^{i\left(k_{x} x_{2}+k_{y} y_{2}\right)} .
$$

Using these Fourier representations, we can express the second integral in Eq. (10) as

$$
\begin{aligned}
& \iint_{-\infty}^{+\infty} d x_{1} d y_{1}\left\langle\alpha_{m i}^{\gamma *}\left(x_{1}, y_{1}\right) \alpha_{n j}^{\gamma}\left(x_{2}, y_{2}\right)\right\rangle \mathrm{G}_{l m}^{*}\left(\mathbf{r}_{0}, x_{1}, y_{1}\right) E_{i}^{*}\left(x_{1}, y_{1}\right) \\
& \quad=\frac{\tilde{\alpha}_{m i}^{\gamma *} \tilde{\alpha}_{n j}^{\gamma}}{4 \pi^{2}} \iint_{-\infty}^{+\infty} d k_{x} d k_{y} \hat{F}_{l m i}^{*}\left(k_{x}, k_{y}\right) \iint_{-\infty}^{+\infty} d k_{x}^{\prime} d k_{y}^{\prime} \mathrm{e}^{-\left(k_{x}^{\prime 2}+k_{y}^{\prime 2}\right) L_{c}^{2} / 4} \mathrm{e}^{i\left(k_{x}^{\prime} x_{2}+k_{y}^{\prime} y_{2}\right)} \iint_{-\infty}^{+\infty} d x_{1} d y_{1} \mathrm{e}^{-i\left[x_{1}\left(k_{x}+k_{x}{ }^{\prime}\right)+y_{1}\left(k_{y}+k_{y}{ }^{\prime}\right)\right]} \\
& \quad=\tilde{\alpha}_{m i}^{\gamma *} \tilde{\alpha}_{n j}^{\gamma} \iint_{-\infty}^{+\infty} d k_{x} d k_{y} \hat{F}_{l m i}^{*}\left(k_{x}, k_{y}\right) \mathrm{e}^{-\left(k_{x}{ }^{2}+k_{y}{ }^{2}\right) L_{c}^{2} / 4} \mathrm{e}^{-i\left(k_{x} x_{2}+k_{y} y_{2}\right)}
\end{aligned}
$$

where we used $\int e^{i(x y)} d x=2 \pi \delta(y)$. The signal (10) can now be calculated as

$$
\begin{aligned}
S\left(\mathbf{r}_{0}\right)= & \frac{\omega_{\mathrm{s}}^{4}}{\varepsilon_{0}^{2} c^{4}} \sum_{l, m, n} \sum_{i, j} \tilde{\alpha}_{m i}^{\gamma *} \tilde{\alpha}_{n j}^{\gamma} \iint_{-\infty}^{+\infty} d k_{x} d k_{y} \hat{F}_{l m i}^{*}\left(k_{x}, k_{y}\right) \mathrm{e}^{-\left(k_{x}{ }^{2}+k_{y}{ }^{2}\right) L_{\mathrm{c}}^{2} / 4} \\
& \times \iint_{-\infty}^{+\infty} d k_{x}^{\prime} d k_{y}^{\prime} \hat{F}_{l n j}\left(k_{x}^{\prime}, k_{y}^{\prime}\right) \iint_{-\infty}^{+\infty} d x_{2} d y_{2} \mathrm{e}^{-i\left[x_{2}\left(k_{x}-k_{x}{ }^{\prime}\right)+y_{2}\left(k_{y}-k_{y}{ }^{\prime}\right)\right]} \\
= & 4 \pi^{2} \frac{\omega_{\mathrm{s}}^{4}}{\varepsilon_{0}^{2} c^{4}} \sum_{l, m, n} \sum_{i, j} \tilde{\alpha}_{m i}^{\gamma *} \tilde{\alpha}_{n j}^{\gamma} \iint_{-\infty}^{+\infty} d k_{x} d k_{y} \hat{F}_{l m i}^{*}\left(k_{x}, k_{y}\right) \hat{F}_{l n j}\left(k_{x}, k_{y}\right) \mathrm{e}^{-\left(k_{x}{ }^{2}+k_{y}{ }^{2}\right) L_{\mathrm{c}}^{2} / 4},
\end{aligned}
$$

which, provided that the Fourier transform (13) can be calculated, is considerably more convenient than the fourfold integral in Eq. (10). In the fully coherent limit $\left(L_{\mathrm{c}} \rightarrow \infty\right)$, there are no statistical variations between points $\mathbf{r}_{1}$ and $\mathbf{r}_{2}$, whereas in the incoherent case $\left(L_{\mathrm{c}} \rightarrow 0\right)$, the response at $\mathbf{r}_{1}$ and $\mathbf{r}_{2}$ is completely uncorrelated and the correlation function reduces to a Dirac delta distribution. In these limiting cases, we find

$$
\begin{aligned}
\lim _{L_{\mathrm{c}} \rightarrow \infty} S\left(\mathbf{r}_{0}\right)= & 16 \pi^{4} \frac{\omega_{\mathrm{s}}^{4}}{\varepsilon_{0}^{2} c^{4}} \sum_{l, m, n} \sum_{i, j} \alpha_{m i}^{\gamma *} \alpha_{n j}^{\gamma} \hat{F}_{l m i}^{*}(0,0) \hat{F}_{l n j}(0,0) \\
= & \frac{\omega_{\mathrm{s}}^{4}}{\varepsilon_{0}^{2} c^{4}} \sum_{l, m, n} \sum_{i, j} \alpha_{m i}^{\gamma *} \alpha_{n j}^{\gamma} \iint_{-\infty}^{+\infty} d x_{1} d y_{1} \mathrm{G}_{l m}^{*}\left(\mathbf{r}_{0}, x_{1}, y_{1}\right) E_{i}^{*}\left(x_{1}, y_{1}\right) \iint_{-\infty}^{+\infty} d x_{2} d y_{2} \mathrm{G}_{l n}\left(\mathbf{r}_{0}, x_{2}, y_{2}\right) E_{j}\left(x_{2}, y_{2}\right) \\
\lim _{L_{\mathrm{c}} \rightarrow 0} S\left(\mathbf{r}_{0}\right) & =4 \pi^{2} \frac{\omega_{\mathrm{s}}^{4}}{\varepsilon_{0}^{2} c^{4}} \sum_{l, m, n} \sum_{i, j} \tilde{\alpha}_{m i}^{\gamma *} \tilde{\alpha}_{n j}^{\gamma} \iint_{-\infty}^{+\infty} d k_{x} d k_{y} \hat{F}_{l m i}^{*}\left(k_{x}, k_{y}\right) \hat{F}_{l n j}\left(k_{x}, k_{y}\right) \\
& =\frac{\omega_{\mathrm{s}}^{4}}{\varepsilon_{0}^{2} c^{4}} \sum_{l, m, n} \sum_{i, j} \tilde{\alpha}_{m i}^{\gamma *} \tilde{\alpha}_{n j}^{\gamma} \iint_{-\infty}^{+\infty} d x d y \mathrm{G}_{l m}^{*}\left(\mathbf{r}_{0}, x, y\right) E_{i}^{*}(x, y) \mathrm{G}_{l n}\left(\mathbf{r}_{0}, x, y\right) E_{j}(x, y)
\end{aligned}
$$


where $\alpha_{m i}^{\gamma *} \alpha_{n j}^{\gamma}=\tilde{\alpha}_{m i}^{\gamma *} \tilde{\alpha}_{n j}^{\gamma} / \pi L_{\mathrm{c}}^{2}$. These two limits were studied in Refs. [25,26] for 1D and 2D systems, respectively. Here, we discuss the more realistic case, where the correlation length $L_{\mathrm{c}}$ has a finite value.

In order to evaluate the signal in Eq. (10), we need to know the exciting field $\mathbf{E}$ at the location $\mathbf{r}=(x, y, 0)$ on the graphene plane. This field corresponds to the superposition of the incident laser field $\mathbf{E}_{0}$ and the localized field generated by the gold tip acting as an optical antenna. It can be represented in terms of a volume integral equation as [27]

$$
\begin{aligned}
\mathbf{E}(\mathbf{r}, \omega)= & \mathbf{E}_{0}(\mathbf{r}, \omega)+\frac{\omega^{2}}{c^{2}} \int d^{3} \mathbf{r}^{\prime \prime} \stackrel{\leftrightarrow}{\mathrm{G}}\left(\mathbf{r}, \mathbf{r}^{\prime \prime}, \omega\right) \\
& \times\left[\varepsilon\left(\mathbf{r}^{\prime \prime}\right)-1\right] \mathbf{E}\left(\mathbf{r}^{\prime \prime}, \omega\right),
\end{aligned}
$$

where $\varepsilon\left(\mathbf{r}^{\prime \prime}, \omega\right)$ is the spatial distribution of the dispersive dielectric constant and $\stackrel{\leftrightarrow}{\mathrm{G}}$ is the Green function of the reference system, which includes the sample, the supporting surface, and the tip. In principle, the field (19) can be substituted into the expression for the signal in Eq. (10), and the signal can be computed numerically. To reduce the numerical complexity, we describe the tip by an anisotropic polarizability [27]

$$
\stackrel{\leftrightarrow}{\alpha}_{\text {tip }}\left(\mathbf{r}^{\prime}\right)=\left[\begin{array}{ccc}
\alpha_{\perp} & 0 & 0 \\
0 & \alpha_{\perp} & 0 \\
0 & 0 & \alpha_{\|}
\end{array}\right],
$$

with the tip axis coinciding with the $z$ direction. $\alpha_{\perp}$ and $\alpha_{\|}$ denote the transverse and longitudinal polarizabilities defined as [27]

$$
\alpha_{\perp}(\omega)=4 \pi \varepsilon_{0} r_{\text {tip }}^{3} \frac{\varepsilon(\omega)-1}{\varepsilon(\omega)+2}
$$

and

$$
\alpha_{\|}(\omega)=2 \pi \varepsilon_{0} r_{\text {tip }}^{3} f_{\mathrm{e}}(\omega),
$$

where $\varepsilon$ denotes the dielectric constant of the tip, $r_{\text {tip }}$ is the tip radius, and $f_{\mathrm{e}}$ is the complex field enhancement factor. Accordingly, a general field $\mathbf{E}$ interacting with the tip induces a dipole $\mathbf{p}_{\text {tip }}=\stackrel{\leftrightarrow}{\alpha}_{\text {tip }} \mathbf{E}$ in the tip. The Green function in Eq. (10) can then be written as

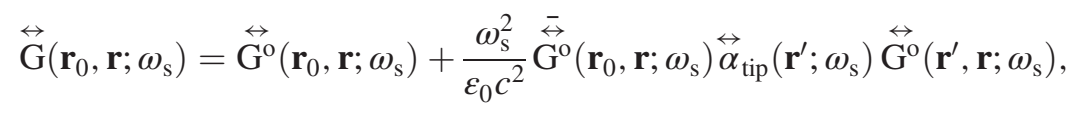

where the first term denotes the free-space propagation from a point $\mathbf{r}=(x, y, 0)$ on the graphene sample to the observation point $\mathbf{r}_{0}$, and the second term corresponds to the interaction with the tip dipole at $\mathbf{r}^{\prime}=(0,0, z)$, that is, free-space propagation from graphene to the tip and subsequent propagation from the tip to the observation point. We apply the same model for the excitation field described by Eq. (19) and obtain

$$
\mathbf{E}(\mathbf{r}, \omega)=\mathbf{E}_{0}(\mathbf{r}, \omega)+\frac{\omega^{2}}{\varepsilon_{0} c^{2}} \overleftrightarrow{\mathrm{G}^{\mathrm{o}}}\left(\mathbf{r}, \mathbf{r}^{\prime} ; \omega\right) \stackrel{\leftrightarrow}{\alpha} \text { tip }(\omega) \mathbf{E}_{0}\left(\mathbf{r}^{\prime}, \omega\right)
$$

Equations (23) and (24) can now be inserted into Eq. (10) to calculate the signal $S\left(\mathbf{r}_{0}, \omega_{\mathrm{s}}\right)$ in terms of the free-space Green function and as a function of the tip position and excitation conditions. Combining Eqs. (23) and (24) gives

$$
\begin{aligned}
& \mathrm{G}_{l n}\left(\mathbf{r}_{0}, x, y ; \omega_{\mathrm{s}}\right) \alpha_{n j}^{\gamma}(x, y ; \omega) E_{j}(x, y, \omega)=\mathrm{G}_{l n}^{\mathrm{o}}\left(\mathbf{r}_{0}, x, y ; \omega_{\mathrm{s}}\right) \alpha_{n j}^{\gamma}(x, y ; \omega) E_{0_{j}}(x, y, \omega) \\
& +\frac{\omega_{\mathrm{s}}^{2}}{\varepsilon_{0} c^{2}}\left[\overleftrightarrow{\mathrm{G}^{\mathrm{o}}}\left(\mathbf{r}_{0}, z ; \omega_{\mathrm{s}}\right) \overleftrightarrow{\alpha}_{\text {tip }}\left(\omega_{\mathrm{s}}\right) \overleftrightarrow{\mathrm{G}^{\mathrm{o}}}\left(z, x, y ; \omega_{\mathrm{s}}\right)\right]_{l n} \alpha_{n j}^{\gamma}(x, y ; \omega) E_{0_{j}}(x, y, \omega) \\
& +\frac{\omega^{2}}{\varepsilon_{0} c^{2}} \mathrm{G}_{l n}^{\mathrm{o}}\left(\mathbf{r}_{0}, x, y ; \omega_{\mathrm{s}}\right) \alpha_{n j}^{\gamma}(x, y ; \omega)\left[\overleftrightarrow{\mathrm{G}^{\mathrm{o}}}(x, y, z ; \omega) \stackrel{\leftrightarrow}{\alpha_{\text {tip }}}(\omega) \mathbf{E}_{0}(z, \omega)\right]_{j} \\
& +\frac{\omega^{2} \omega_{\mathrm{s}}^{2}}{\varepsilon_{0}^{2} c^{4}}\left[\stackrel{\leftrightarrow}{\mathrm{G}^{\mathrm{o}}}\left(\mathbf{r}_{0}, z ; \omega_{\mathrm{s}}\right) \stackrel{\leftrightarrow}{\alpha_{\text {tip }}}\left(\omega_{\mathrm{s}}\right) \overleftrightarrow{\mathrm{G}^{\mathrm{o}}}\left(z, x, y ; \omega_{\mathrm{s}}\right)\right]_{l n} \\
& \times \alpha_{n j}^{\gamma}(x, y ; \omega)\left[\overleftrightarrow{\mathrm{G}^{\mathrm{o}}}(x, y, z ; \omega) \stackrel{\leftrightarrow}{\alpha_{\text {tip }}}(\omega) \mathbf{E}_{0}(z, \omega)\right]_{j},
\end{aligned}
$$

where we explicitly used $\mathbf{r}^{\prime}=(0,0, z)$ and $\mathbf{r}=(x, y, 0)$. Since the polarizability along the tip axis is much larger than transverse to it $\left(\left|\alpha_{\|}\right| \gg\left|\alpha_{\perp}\right|\right)$, we neglect $\alpha_{\perp}$ in Eqs. (20) and (25) and obtain 


$$
\begin{aligned}
\mathrm{G}_{l n}\left(\mathbf{r}_{0}, x, y ; \omega_{\mathrm{s}}\right) \alpha_{n j}^{\gamma}(x, y, \omega) E_{j}(x, y, \omega)= & \mathrm{G}_{l n}^{\mathrm{o}}\left(\mathbf{r}_{0}, x, y ; \omega_{\mathrm{s}}\right) \alpha_{n j}^{\gamma}(x, y, \omega) E_{0 j}(x, y, \omega) \\
& +\frac{\omega_{\mathrm{s}}^{2}}{\varepsilon_{0} c^{2}} \mathrm{G}_{l z}^{\mathrm{o}}\left(\mathbf{r}_{0}, z^{\prime} ; \omega_{\mathrm{s}}\right) \alpha_{\|}\left(\omega_{\mathrm{s}}\right) \mathrm{G}_{z n}^{\mathrm{o}}\left(z^{\prime}, x, y ; \omega_{\mathrm{s}}\right) \alpha_{n j}^{\gamma}(x, y, \omega) E_{0 j}(x, y, \omega) \\
& +\frac{\omega^{2}}{\varepsilon_{0} c^{2}} \mathrm{G}_{l n}^{\mathrm{o}}\left(\mathbf{r}_{0}, x, y ; \omega_{\mathrm{s}}\right) \alpha_{n j}^{\gamma}(x, y, \omega) \mathrm{G}_{j z}^{\mathrm{o}}\left(x, y, z^{\prime} ; \omega\right) \alpha_{\|}(\omega) E_{0 z}\left(z^{\prime}, \omega\right) \\
& +\frac{\omega^{2} \omega_{\mathrm{s}}^{2}}{\varepsilon_{0}^{2} c^{4}} \mathrm{G}_{l z}^{\mathrm{o}}\left(\mathbf{r}_{0}, z^{\prime} ; \omega_{\mathrm{s}}\right) \alpha_{\|}\left(\omega_{\mathrm{s}}\right) \mathrm{G}_{z n}^{\mathrm{o}}\left(z^{\prime}, x, y ; \omega_{\mathrm{s}}\right) \alpha_{n j}^{\gamma}(x, y, \omega) \\
& \times \mathrm{G}_{j z}^{\mathrm{o}}\left(x, y, z^{\prime} ; \omega\right) \alpha_{\|}(\omega) E_{0 z}\left(z^{\prime}, \omega\right) .
\end{aligned}
$$

The first term in Eq. (26) is the interaction of the incident field with the sample (S), the second term accounts for the incident field that scatters at the sample and then at the tip (TS), the third term is the interaction with the tip and then with the sample (ST), and the last term is the interaction with the tip, then the sample and the tip again (TST). In other words, Eq. (26) describes the following interaction series [28]:

$$
\mathbf{S}+\mathbf{T S}+\mathbf{S T}+\mathbf{T S T}
$$

which is illustrated in Fig. 3. In our scheme, the $\mathbf{S}$ term in Eq. (26) describes standard far-field Raman scattering (e.g., confocal Raman scattering), and the TST accounts for standard tip-enhanced Raman scattering (TERS). The terms ST and TS originate from the interference between the near field and the far field. We have suppressed higher-order interaction terms between the graphene sample and the tip
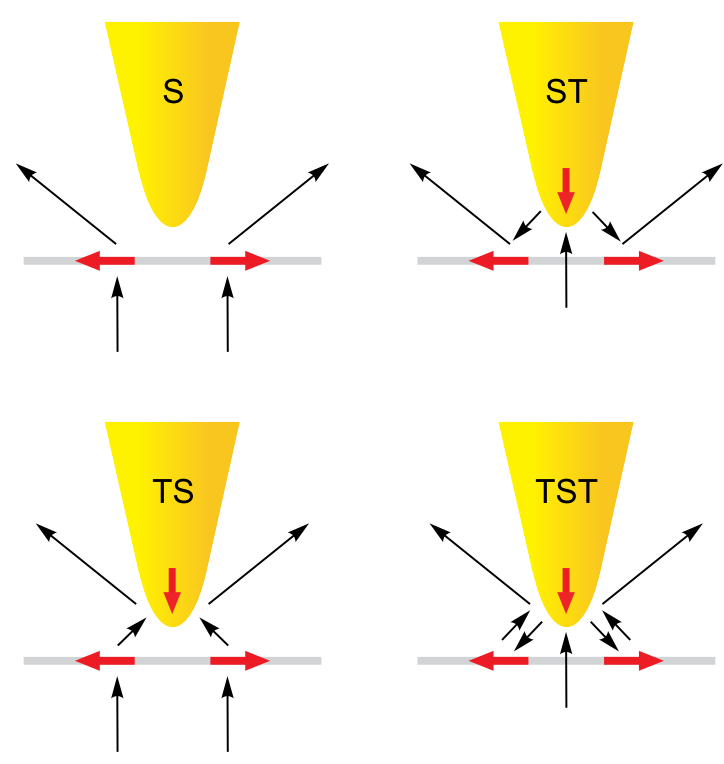

FIG. 3. Interaction series in TERS. Red arrows represent induced dipoles; black arrows indicate electromagnetic interactions. because graphene interacts only weakly with light (3\% absorption). TERS relies on excitation fields that exhibit a strong polarization component along the tip axis. Such conditions can be provided, for example, by a focused radially polarized laser beam incident along the tip axis [29]. Because the $z$ component of the incident field is much larger than the in-plane $x, y$ components, the signal strength generated by the TS component is roughly 10 times weaker than by the ST term. For this reason, we neglect the TS term in the present study.

We now have all the ingredients to evaluate the TST and ST components of the near-field Raman signal for 2D and 1D samples.

$T S T / 2 D$. - In the following, we proceed with the evaluation of the $S^{\mathrm{TST}}\left(\mathbf{r}_{0}\right)$ signal generated by the TST component for the 2D modes $\left(G\right.$ and $\left.G^{\prime}\right)$. We assume that the exciting field is homogeneous, that is, $E_{0 z}(z, \omega)=E_{0 z}(\omega)$, which is fulfilled for tip-sample distances $z$ much smaller than $\lambda$. Considering the TST term in Eq. (26), we can express the Fourier transform of $\mathrm{G}_{l n}\left(\mathbf{r}_{0}, x, y ; \omega_{\mathrm{s}}\right) E_{j}(x, y, \omega)$ [Eq. (13)] as

$$
\begin{aligned}
\hat{F}_{l n j}\left(k_{x}, k_{y}\right)= & \frac{1}{4 \pi^{2}} \frac{\omega^{2} \omega_{\mathrm{s}}^{2}}{\varepsilon_{0}^{2} c^{4}} \alpha_{\|}(\omega) \alpha_{\|}\left(\omega_{\mathrm{s}}\right) \mathrm{G}_{l z}^{\mathrm{o}}\left(\mathbf{r}_{0}, z ; \omega_{\mathrm{s}}\right) E_{0 z}(\omega) \\
& \times \iint_{-\infty}^{+\infty} d x d y \mathrm{G}_{z n}^{\mathrm{o}}\left(x, y, z ; \omega_{\mathrm{s}}\right) \\
& \times \mathrm{G}_{j z}^{\mathrm{o}}(x, y, z ; \omega) \mathrm{e}^{-i\left(k_{x} x+k_{y} y\right)}
\end{aligned}
$$

Because of the short-range interaction between tip and sample $\left(R=\left|\mathbf{r}-\mathbf{r}^{\prime}\right| \ll \lambda, \lambda_{\mathrm{s}}\right)$, we retain only the nonretarded near-field term in the Green functions $\mathrm{G}_{z n}^{\mathrm{o}}$ and $\mathrm{G}_{j z}^{\mathrm{o}}$, that is,

$\mathrm{G}_{z n}^{\mathrm{o}}\left(\omega_{\mathrm{s}}\right)=\frac{1}{4 \pi k_{\mathrm{s}}^{2}} \frac{3 z n}{R^{5}} \quad$ and $\quad \mathrm{G}_{j z}^{\mathrm{o}}(\omega)=\frac{1}{4 \pi k^{2}} \frac{3 j z}{R^{5}}$

where $k_{\mathrm{s}}=\omega_{\mathrm{s}} / c$ and $k=\omega / c$. The integral in Eq. (28) now becomes 


$$
\begin{aligned}
& \iint_{-\infty}^{+\infty} d x d y \mathrm{G}_{z n}^{\mathrm{o}}\left(x, y, z ; \omega_{\mathrm{s}}\right) \mathrm{G}_{j z}^{\mathrm{o}}(x, y, z ; \omega) \mathrm{e}^{-i\left(k_{x} x+k_{y} y\right)} \\
& =\frac{9}{16 \pi^{2}} \frac{c^{4}}{\omega_{\mathrm{s}}^{2} \omega^{2}} \iint_{-\infty}^{+\infty} d x d y \frac{n j z^{2}}{\left(x^{2}+y^{2}+z^{2}\right)^{5}} \mathrm{e}^{-i\left(k_{x} x+k_{y} y\right)} .
\end{aligned}
$$

This integral has a complicated analytical form, and hence it is convenient to approximate the integrand by a superposition of exponential functions,

$$
\begin{aligned}
& \frac{\left(n j / z^{2}\right)}{\left.z^{6}[(x / z)]^{2}+(y / z)^{2}+1\right]^{5}} \\
& \approx \frac{\left(n j / z^{2}\right)}{z^{6}}\left[a_{0} \mathrm{e}^{-b_{0}\left(x^{2}+y^{2}\right) / z^{2}}+c_{0} \mathrm{e}^{-d_{0}\left(x^{2}+y^{2}\right) / z^{2}}\right],
\end{aligned}
$$

where $a_{0}, b_{0}, c_{0}$, and $d_{0}$ are fitting constants. Figure 4 shows a comparison of the original function and the approximation. The fitting constants of the latter are given in the caption. The agreement is very good, and the approximation is not expected to have any influence on the results of this study.

The Fourier transform (28) is now calculated as

$$
\begin{aligned}
\hat{F}_{l n j}\left(k_{x}, k_{y}\right)= & \frac{9}{256 \pi^{3} \varepsilon_{0}^{2} z^{4}} \alpha_{\|}(\omega) \alpha_{\|}\left(\omega_{\mathrm{s}}\right) \\
& \times \mathrm{G}_{l z}^{\mathrm{o}}\left(\mathbf{r}_{0}, z ; \omega_{\mathrm{s}}\right) E_{0 z}(z, \omega) \hat{h}_{n j}\left(k_{x}, k_{y} ; z\right),
\end{aligned}
$$

with

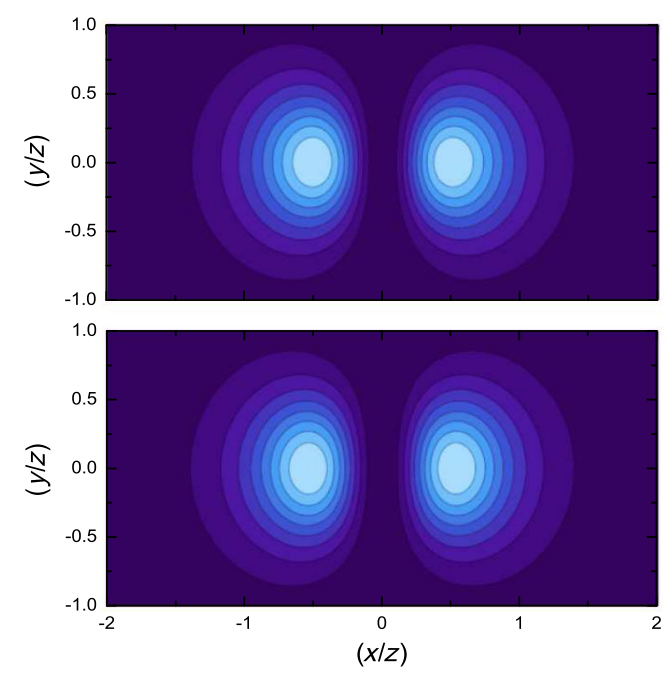

$$
\begin{aligned}
\hat{h}_{n j}\left(k_{x}, k_{y} ; z\right)= & {\left[\frac{a_{0}\left(2 \delta_{n j} b_{0}-k_{n} k_{j} z^{2}\right)}{b_{0}^{3}} \mathrm{e}^{-\left(k_{x}^{2}+k_{y}^{2}\right) z^{2} / 4 b_{0}}\right.} \\
& \left.+\frac{c_{0}\left(2 \delta_{n j} d_{0}-k_{n} k_{j} z^{2}\right)}{d_{0}^{3}} \mathrm{e}^{-\left(k_{x}^{2}+k_{y}^{2}\right) z^{2} / 4 d_{0}}\right],
\end{aligned}
$$

$\delta_{n j}$ being a Kronecker delta. To calculate the Raman signal (16) for the TST interaction term, we define the expressions

$$
\begin{aligned}
f_{m i, n j}\left(z, L_{\mathrm{c}}\right)= & \iint_{-\infty}^{+\infty} d k_{x} d k_{y} \hat{h}_{m i}^{*}\left(k_{x}, k_{y} ; z\right) \\
& \times \hat{h}_{n j}\left(k_{x}, k_{y} ; z\right) \mathrm{e}^{-\left(k_{x}^{2}+k_{y}^{2}\right) L_{\mathrm{c}}^{2} / 4},
\end{aligned}
$$

which can be calculated analytically using the result in Eq. (33). The functions $f_{m i, n j}$ have the properties

$f_{m i, n j}\left(z, L_{\mathrm{c}} \rightarrow \infty\right)=1 / L_{\mathrm{c}}^{2}, \quad f_{m i, n j}\left(z, L_{\mathrm{c}} \rightarrow 0\right)=1 / z^{2}$.

Inserting Eq. (34) into Eq. (16) yields

$$
\begin{aligned}
S^{\mathrm{TST}}\left(\mathbf{r}_{0}\right)= & \frac{(3 / 8)^{4}}{4 \pi^{4}} \frac{\omega_{\mathrm{s}}^{4}}{\varepsilon_{0}^{6} c^{4} z^{8}} \sum_{l} \mid \alpha_{\|}(\omega) \alpha_{\|}\left(\omega_{\mathrm{s}}\right) \\
& \times\left.\mathrm{G}_{l z}^{\mathrm{o}}\left(\mathbf{r}_{0}, z ; \omega_{\mathrm{s}}\right) E_{0 z}(z, \omega)\right|^{2} \\
& \times\left[\sum_{m, n} \sum_{i, j} \tilde{\alpha}_{m i}^{\gamma *} \tilde{\alpha}_{n j}^{\gamma} f_{m i, n j}\left(z, L_{\mathrm{c}}\right)\right] .
\end{aligned}
$$

Thus, we find that for graphene with zero correlation length $\left(L_{\mathrm{c}} \rightarrow 0\right)$, the signal decays as $z^{-10}$ from the graphene sample, whereas for graphene with infinite correlation length $\left(L_{\mathrm{c}} \rightarrow \infty\right)$, it decays as $z^{-8}$, consistent with the theory described in Ref. [26].

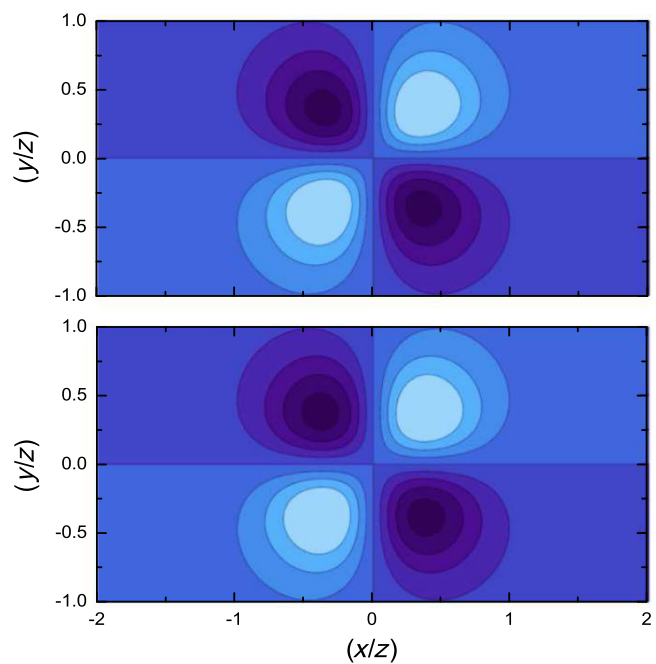

FIG. 4. Comparison of original function (top panels) and approximation (bottom panels) according to Eq. (31). The left panels correspond to $n=j$ ( $=x$ in this case), while the right panels correspond to $n \neq j$. In both cases, the following fitting constants were used: $a_{0}=0.74, b_{0}=4.0, c_{0}=0.08$, and $d_{0}=1.5$. 
For the modes with $\mathrm{E}_{2 \mathrm{~g} 1}$ and $\mathrm{A}_{1}$ symmetries, for which the only non-null terms in the respective Raman tensors are $\tilde{\alpha}_{x x}^{\gamma}$ and $\tilde{\alpha}_{y y}^{\gamma}$ [see Eq. (9)], the signal $S\left(\mathbf{r}_{0}\right)$ is given as

$$
\begin{aligned}
S^{\mathrm{TST}}\left(\mathbf{r}_{0}\right)\left(\mathrm{E}_{2 \mathrm{~g} 1}, \mathrm{~A}_{1}\right)= & \frac{(3 / 8)^{4}}{4 \pi^{4} \varepsilon_{0}^{4} z^{8}} \frac{\omega_{\mathrm{s}}^{4}}{\varepsilon_{0}^{2} c^{4}} \sum_{l}\left|\alpha_{\|}(\omega) \alpha_{\|}\left(\omega_{\mathrm{s}}\right) \mathrm{G}_{l z}^{\mathrm{o}}\left(\mathbf{r}_{0}, z ; \omega_{\mathrm{s}}\right) E_{0 z}(z, \omega)\right|^{2} \\
& \times\left(\left[\tilde{\alpha}_{x x}^{\gamma *} \tilde{x}_{x x}^{\gamma}+\tilde{\alpha}_{y y}^{\gamma *} \tilde{\alpha}_{y y}^{\gamma}\right] f_{x x, x x}\left(z, L_{\mathrm{c}}\right)+2 \operatorname{Re}\left[\tilde{\alpha}_{x x}^{\gamma *} \tilde{\alpha}_{y y}^{\gamma} f_{x x, y y}\left(z, L_{\mathrm{c}}\right)\right]\right),
\end{aligned}
$$

where we have used the properties $f_{x x, x x}\left(z, L_{\mathrm{c}}\right)=f_{y y, y y}\left(z, L_{\mathrm{c}}\right)$ and $f_{x x, y y}\left(z, L_{\mathrm{c}}\right)=f_{y y, x x}\left(z, L_{\mathrm{c}}\right)$. On the other hand, for modes belonging to the $\mathrm{E}_{2 \mathrm{~g} 2}$ irreducible representation, for which $\tilde{\alpha}_{x y}^{\gamma}=\tilde{\alpha}_{y x}^{\gamma} \neq 0$ [see Eq. (9)], we have

$$
S^{\mathrm{TST}}\left(\mathbf{r}_{0}\right)\left(\mathrm{E}_{2 \mathrm{~g} 2}\right)=\frac{(3 / 8)^{4}}{4 \pi^{4} \varepsilon_{0}^{4} z^{8}} \frac{\omega_{\mathrm{s}}^{4}}{\varepsilon_{0}^{2} c^{4}} \sum_{l}\left|\alpha_{\|}(\omega) \alpha_{\|}\left(\omega_{\mathrm{s}}\right) \mathrm{G}_{l z}^{\mathrm{o}}\left(\mathbf{r}_{0}, z ; \omega_{\mathrm{s}}\right) E_{0 z}(z, \omega)\right|^{2} 4 \operatorname{Re}\left[\tilde{\alpha}_{x y}^{\gamma *} \tilde{\alpha}_{y x}^{\gamma} f_{x y, y x}\left(z, L_{\mathrm{c}}\right)\right],
$$

where we have used the fact that $f_{x y, y x}\left(z, L_{\mathrm{c}}\right)=f_{y x, x y}\left(z, L_{\mathrm{c}}\right)=f_{x y, x y}\left(z, L_{\mathrm{c}}\right)=f_{y x, y x}\left(z, L_{\mathrm{c}}\right)$.

Next, we rewrite the tip polarizability component $\alpha_{\|}(\omega)$ in terms of the complex field enhancement factor $f_{\mathrm{e}}(\omega)$ and the tip radius $r_{\text {tip }}$ [Eq. (22)], and insert the values of the polarizability tensors (9) in Eqs. (37) and (38). For the G band, we find

$$
S_{\mathrm{G}}^{\mathrm{TST}}\left(\mathbf{r}_{0}\right)=\frac{81}{512} \frac{\omega_{\mathrm{s}}^{4}}{\varepsilon_{0}^{2} c^{4}} \frac{r_{\text {tip }}^{12} \tilde{f}_{\mathrm{e}}(\omega)^{4}}{z^{8}} \sum_{l}\left|\mathrm{G}_{l z}^{\mathrm{o}}\left(\mathbf{r}_{0}, z ; \omega_{\mathrm{s}}\right) E_{0 z}(z, \omega) \tilde{\alpha}^{\mathrm{G}}\left(\omega_{\mathrm{s}} ; \omega\right)\right|^{2}\left[f_{x x, x x}\left(z, L_{\mathrm{c}}\right)-f_{x x, y y}\left(z, L_{\mathrm{c}}\right)+2 f_{x y, y x}\left(z, L_{\mathrm{c}}\right)\right],
$$

where we have considered $\tilde{f}_{\mathrm{e}}(\omega) \approx \tilde{f}_{\mathrm{e}}\left(\omega_{s}\right), \tilde{f}_{\mathrm{e}}$ being the real part of $f_{\mathrm{e}}$. For the Raman $\mathrm{G}^{\prime}$ band, we obtain

$$
S_{\mathrm{G}^{\prime}}^{\mathrm{TST}}\left(\mathbf{r}_{0}\right)=\frac{81}{512} \frac{\omega_{\mathrm{s}}^{4}}{\varepsilon_{0}^{2} c^{4}} \frac{r_{\text {tip }}^{12} \tilde{f}_{\mathrm{e}}(\omega)^{4}}{z^{8}} \sum_{l}\left|\mathrm{G}_{l z}^{\mathrm{o}}\left(\mathbf{r}_{0}, z ; \omega_{\mathrm{s}}\right) E_{0 z}(z, \omega) \tilde{\alpha}^{\mathrm{G}^{\prime}}\left(\omega_{\mathrm{s}} ; \omega\right)\right|^{2}\left[f_{x x, x x}\left(z, L_{\mathrm{c}}\right)+f_{x x, y y}\left(z, L_{\mathrm{c}}\right)\right] .
$$

The dependence on the correlation length $L_{\mathrm{c}}$ and the distance $z$ is solely determined by the last terms in the expressions. The first terms only account for the overall strength of the signal. They depend on the Raman cross section, the local field enhancement, the tip radius, and the detection conditions. In the following, we will discuss the consequences of the different terms in Eqs. (39) and (40).

In Fig. 5, we plot the distance dependence of the $\mathrm{G}$ and $\mathrm{G}^{\prime}$ TST signals [according to Eqs. (39) and (40), respectively] for different correlation lengths $L_{\mathrm{c}}$. For all cases, we set $\tilde{f}_{\mathrm{e}}(\omega)=3$ and $r_{\text {tip }}=15 \mathrm{~nm}$. Considering the minimum distance between the tip apex and the sample surface to be $5 \mathrm{~nm}$ (this value is determined by the set point that controls the tip-sample distance in TERS experiments), the shortest distance $z_{\min }$ between the graphene surface and the tip dipole becomes $z_{\min }=20 \mathrm{~nm}$. The curves coincide for $L_{\mathrm{c}}=0$, which corresponds to the case where there is no correlation between neighboring graphene lattice points. In this case, the Raman signal is added up incoherently; that is, the intensities of neighboring lattice points are summed up. For a finite correlation length $L_{\mathrm{c}}$, we observe that the distance curves for the $\mathrm{G}$ and $\mathrm{G}^{\prime}$ bands become different, with the $\mathrm{G}$ band showing a markedly weaker distance dependence. This deviation arises from the coherent interaction between neighboring lattice points which are symmetric with respect to the lateral location of the tip. As $L_{\mathrm{c}}$ increases, the distance dependence of the $\mathrm{G}^{\prime}$ band transits from $z^{-10}$ to $z^{-8}$. The predictions shown in
Fig. 5 indicate that the phonon correlation length in graphene can be experimentally determined by measuring the $\mathrm{G}$ and $\mathrm{G}^{\prime}$ approach curves.

The interference effects that generate different enhancements for the $G$ and $G^{\prime}$ bands at finite values of $L_{\mathrm{c}}$ are illustrated in Fig. 6. The yellow spot represents the top view of the tip (axis along the $z$ direction) with the graphene lattice underneath (lying in the $x y$ plane). In (a), the $\otimes$ symbol represents the tip dipole induced by the incident field $\mathbf{E}_{0}(z, \omega)$. The green arrows represent the $x$ and $y$ inplane components of the electric field $\mathbf{E}(\mathbf{r} ; \omega)$ at the graphene lattice, generated by the tip dipole. The respective induced Raman dipoles $\mathbf{p}^{\gamma}\left(\mathbf{r} ; \omega_{\mathrm{s}}\right)$ [Eq. (4)] in the graphene plane are represented by the red arrows in (b-d) for the modes with $\mathrm{A}_{1}, \mathrm{E}_{2 \mathrm{~g} 1}$, and $\mathrm{E}_{2 \mathrm{~g} 2}$ symmetries, respectively. The directions of the induced Raman dipoles are determined by the Raman polarizability tensors $\stackrel{\leftrightarrow}{\alpha}\left(\mathbf{r} ; \omega_{\mathrm{s}}, \omega\right)$ in Eq. (9). By considering the fully coherent case $\left(L_{\mathrm{c}} \rightarrow \infty\right)$, the scattered field generated by the $\mathrm{A}_{1}$ Raman dipoles add constructively at the tip apex, generating a strong induced dipole at the tip [represented by the $\otimes$ symbol in (b)]. On the other hand, the field generated by the Raman dipoles interferes destructively at the tip apex for the $E_{2 g 1}$ and $E_{2 g 2}$ symmetries [(c) and (d), respectively]. For finite $L_{\mathrm{c}}$, the $\mathrm{G}$ band $\left(E_{2 g}\right.$ symmetry) TST signal will not be exactly null, but it will be clearly weaker than in the $G^{\prime}$ case $\left(A_{1}\right.$ symmetry). Notice that these two distinct situations are solely associated with the symmetry of the Raman modes. 

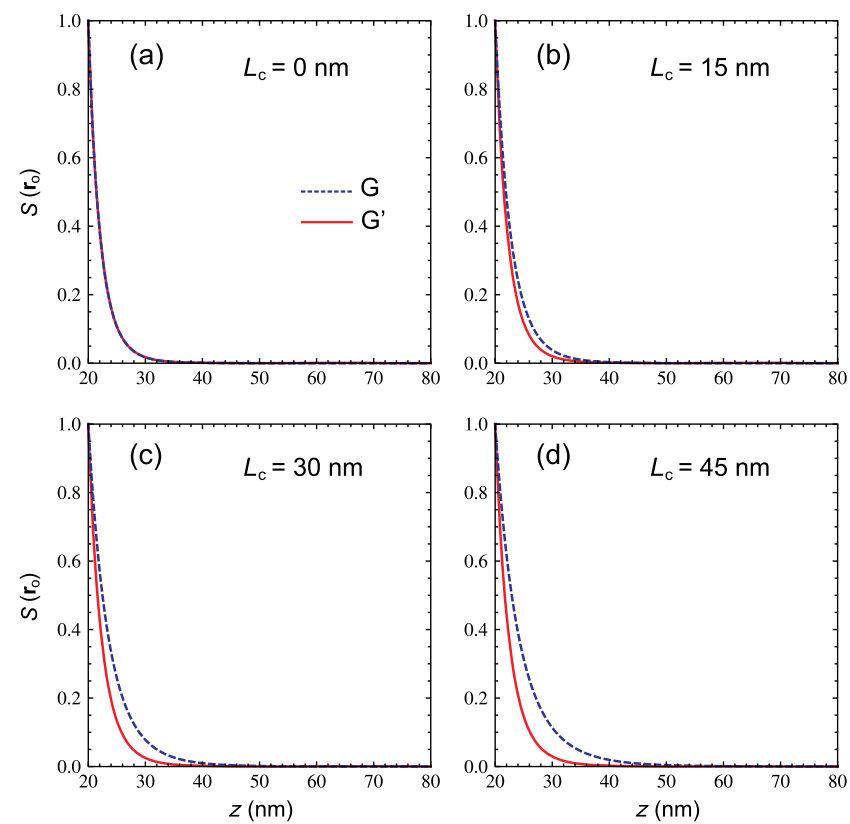

FIG. 5. Distance dependence of the TST signal for the $\mathrm{G}$ and $\mathrm{G}^{\prime}$ bands. Panels (a-d) are evaluated for different correlation lengths $L_{\mathrm{c}}: 0,15,30$, and $45 \mathrm{~nm}$, respectively. In all cases, we used $\tilde{f}_{\mathrm{e}}=3$. The signal $S\left(\mathbf{r}_{0}\right)$ is normalized to 1 for $z_{\min }=20 \mathrm{~nm}$, which corresponds to the closest tip-sample distance for $r_{\text {tip }}=15 \mathrm{~nm}$, considering that the minimal distance between the tip apex and the sample is about $5 \mathrm{~nm}$. For $L_{\mathrm{c}}=0$, the curves for $\mathrm{G}$ and $\mathrm{G}^{\prime}$ coincide. This case corresponds to an incoherent addition of the Raman response in different points of the graphene lattice. As $L_{c}$ increases, interferences between neighboring lattice points which are symmetric with respect to the lateral location of the tip give rise to a distance dependence that is different for the $\mathrm{G}$ and $\mathrm{G}^{\prime}$ bands.
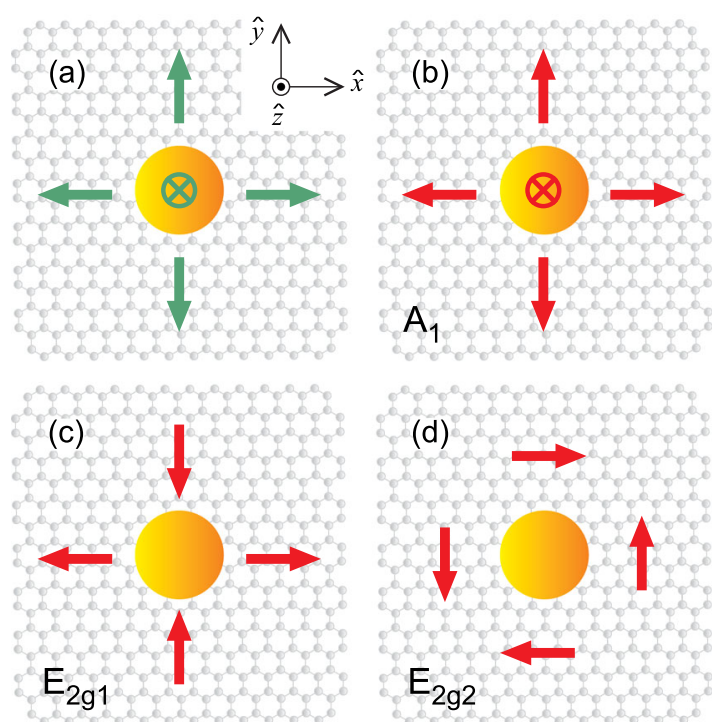

FIG. 6. Symmetries of near-field Raman scattering in the TST configuration. The yellow spots represent the top view of the tip (axis along the $z$ direction) with the graphene lattice underneath (lying in the $x y$ plane). In (a), the $\otimes$ symbol represents the tip dipole induced by the incident field. The green arrows represent the $x$ and $y$ in-plane components of the electric field generated by the tip dipole at the graphene lattice. The respective induced Raman dipoles in the graphene plane are represented by the red arrows in (b-d) for the modes with $\mathrm{A}_{1}, \mathrm{E}_{2 \mathrm{~g} 1}$, and $\mathrm{E}_{2 \mathrm{~g} 2}$ symmetries, respectively. By considering the fully coherent case $\left(L_{\mathrm{c}} \rightarrow \infty\right)$, the scattered fields generated by the $\mathrm{A}_{1}$ Raman dipoles add constructively at the tip apex, generating a strong induced dipole at the tip [represented by the $\otimes$ symbol in (b)]. On the other hand, the field generated by the Raman dipoles interfere destructively at the tip apex for the $E_{2 \mathrm{~g} 1}$ and $\mathrm{E}_{2 \mathrm{~g} 2}$ symmetries [(c) and (d), respectively].

ST/2D.—Now, we evaluate the ST component [third term in Eq. (26)] for the 2D modes (G and $\left.\mathrm{G}^{\prime}\right)$. From Eq. (16), the Raman intensity in the ST configuration can be calculated as

$$
S^{\mathrm{ST}}\left(\mathbf{r}_{0}\right)=4 \pi^{2} \frac{\omega_{\mathrm{s}}^{4}}{\varepsilon_{0}^{2} c^{4}} \sum_{l, m, n} \sum_{i, j} \tilde{\alpha}_{m i}^{\gamma *} \tilde{\alpha}_{n j}^{\gamma} \iint_{-\infty}^{+\infty} d k_{x} d k_{y} \hat{F}_{l m i}^{*}\left(k_{x}, k_{y}\right) \hat{F}_{l n j}\left(k_{x}, k_{y}\right) \mathrm{e}^{-\left(k_{x}{ }^{2}+k_{y}{ }^{2}\right) L_{\mathrm{c}}^{2} / 4},
$$

where, according to Eq. (26), the $\hat{F}_{l n j}^{*}\left(k_{x}, k_{y}\right)$ function (28) in the $\mathbf{S T}$ configuration assumes the form

$$
\begin{aligned}
\hat{F}_{l n j}\left(k_{x}, k_{y}\right) & =\frac{1}{4 \pi^{2}} \frac{\omega^{2}}{\varepsilon_{0} c^{2}} \alpha_{\|}(\omega) \mathrm{G}_{l n}^{\mathrm{o}}\left(\mathbf{r}_{0}, z ; \omega_{\mathrm{s}}\right) E_{0 z}(z, \omega) \iint_{-\infty}^{+\infty} d x d y \mathrm{G}_{j z}^{\mathrm{o}}(x, y, z ; \omega) \mathrm{e}^{-i\left(k_{x} x+k_{y} y\right)} \\
& =\frac{3}{16 \pi^{3} \varepsilon_{0}} \alpha_{\|}(\omega) \mathrm{G}_{l n}^{\mathrm{o}}\left(\mathbf{r}_{0}, z ; \omega_{\mathrm{s}}\right) E_{0 z}(z, \omega) \iint_{-\infty}^{+\infty} d x d y \frac{j z}{\left(x^{2}+y^{2}+z^{2}\right)^{(5 / 2)}} \mathrm{e}^{-i\left(k_{x} x+k_{y} y\right)}
\end{aligned}
$$

As before, the integrand in Eq. (42) can be approximated as

$$
\frac{j z}{\left(x^{2}+y^{2}+z^{2}\right)^{(5 / 2)}} \approx \frac{j}{z^{4}}\left[a_{0}^{\prime} \mathrm{e}^{-b_{0}^{\prime}\left(x^{2}+y^{2}\right) / z^{2}}+c_{0}^{\prime} \mathrm{e}^{-d_{0}^{\prime}\left(x^{2}+y^{2}\right) / z^{2}}\right]
$$

with $a_{0}^{\prime}, b_{0}^{\prime}, c_{0}^{\prime}$, and $d_{0}^{\prime}$ being fitting constants. Figure 7 shows a comparison of the original function and the approximation. 


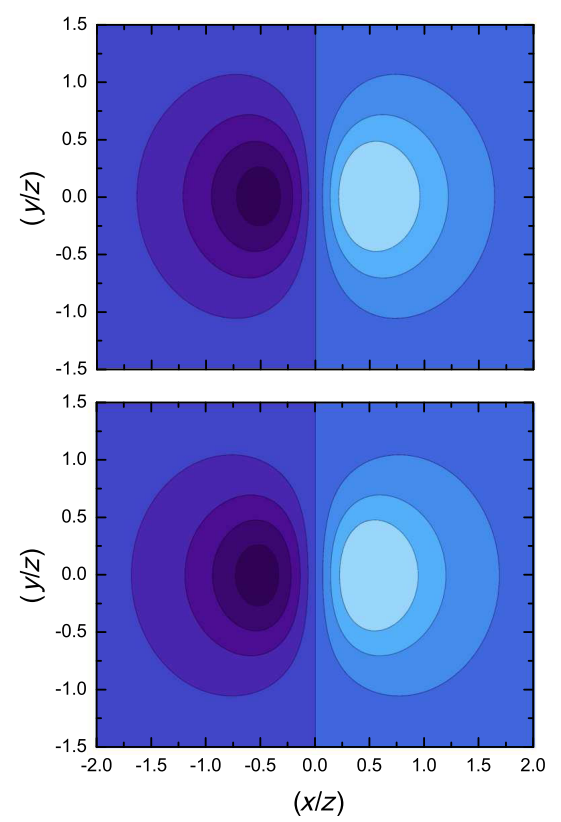

FIG. 7. Comparison of original function (top panel) and approximation (bottom panel) according to Eq. (43), for $j=x$. The following fitting constants were used: $a_{0}^{\prime}=0.78, b_{0}^{\prime}=2.4$, $c_{0}^{\prime}=0.18$, and $d_{0}^{\prime}=0.56$.

The Fourier transform (42) can now be evaluated as

$$
\begin{aligned}
\hat{F}_{l n j}\left(k_{x}, k_{y}\right)= & \frac{(-i) 3}{32 \pi^{2} \varepsilon_{0}} \alpha_{\|}(\omega) \mathrm{G}_{l n}^{\mathrm{o}}\left(\mathbf{r}_{0}, z ; \omega_{\mathrm{s}}\right) E_{0 z}(z, \omega) \\
& \times \hat{h}_{j}\left(k_{x}, k_{y} ; z\right),
\end{aligned}
$$

with

$$
\hat{h}_{j}\left(k_{x}, k_{y} ; z\right)=k_{j}\left[\frac{a_{0}^{\prime}}{b_{0}^{\prime 2}} \mathrm{e}^{-\left(k_{x}^{2}+k_{y}^{2}\right) z^{2} / 4 b_{0}^{\prime}}+\frac{c_{0}^{\prime}}{d_{0}^{\prime 2}} \mathrm{e}^{-\left(k_{x}^{2}+k_{y}^{2}\right) z^{2} / 4 d_{0}^{\prime}}\right] .
$$

To calculate the Raman signal (41) for the $\mathbf{S T}$ interaction term, we define the expressions

$$
\begin{aligned}
\ell_{i j}\left(z, L_{\mathrm{c}}\right)= & \iint_{-\infty}^{+\infty} d k_{x} d k_{y} \hat{h}_{i}^{*}\left(k_{x}, k_{y} ; z\right) \hat{h}_{j}\left(k_{x}, k_{y} ; z\right) \\
& \times \mathrm{e}^{-\left(k_{x}{ }^{2}+k_{y}{ }^{2}\right) L_{\mathrm{c}}^{2} / 4},
\end{aligned}
$$

which can be solved analytically. They have the properties

$$
\ell_{i j}\left(z, L_{\mathrm{c}} \rightarrow \infty\right)=1 / L_{\mathrm{c}}^{4}, \quad \ell_{i j}\left(z, L_{\mathrm{c}} \rightarrow 0\right)=1 / z^{4} .
$$

Inserting Eqs. (44)-(46) into Eq. (41) yields

$$
\begin{aligned}
S^{\mathrm{ST}}\left(\mathbf{r}_{0}\right)= & \frac{9}{256 \pi^{2}} \frac{\omega_{\mathrm{s}}^{4}}{\varepsilon_{0}^{4} c^{4}} \sum_{l, m, n} \sum_{i}\left[\mathrm{G}_{l m}^{\mathrm{o} *}\left(\mathbf{r}_{0}, z ; \omega_{\mathrm{s}}\right) \mathrm{G}_{l n}^{\mathrm{o}}\left(\mathbf{r}_{0}, z ; \omega_{\mathrm{s}}\right)\right. \\
& \left.\times\left|\alpha_{\|}(\omega) E_{0 z}(z, \omega)\right|^{2}\right]\left[\tilde{\alpha}_{m i}^{\gamma *} \tilde{\alpha}_{n i}^{\gamma} \ell_{i i}\left(z, L_{\mathrm{c}}\right)\right]
\end{aligned}
$$

where we have used the properties $\ell_{x x}\left(z, L_{\mathrm{c}}\right)=$ $\ell_{y y}\left(z, L_{\mathrm{c}}\right) \neq 0$ and $\ell_{x y}\left(z, L_{\mathrm{c}}\right)=\ell_{y x}\left(z, L_{\mathrm{c}}\right)=0$. For the modes with $\mathrm{E}_{2 \mathrm{~g} 1}$ and $\mathrm{A}_{1}$ symmetries, the $\mathbf{S T}$ component of the signal is given by

$$
\begin{aligned}
S^{\mathrm{ST}}\left(\mathbf{r}_{0}\right)\left(\mathrm{E}_{2 \mathrm{~g} 1}, \mathrm{~A}_{1}\right)= & \frac{9}{64} \frac{\omega_{\mathrm{s}}^{4}}{\varepsilon_{0}^{4} c^{4}} \sum_{l} \mid \mathrm{G}_{l x}^{\mathrm{o}}\left(\mathbf{r}_{0}, z ; \omega_{\mathrm{s}}\right) \alpha_{\|}(\omega) \\
& \times\left. E_{0 z}(z, \omega)\right|^{2}\left(\tilde{\alpha}_{x x}^{\gamma *} \tilde{\alpha}_{x x}^{\gamma}+\tilde{\alpha}_{y y}^{\gamma *} \tilde{\alpha}_{y y}^{\gamma}\right) \\
& \times \ell_{x x}\left(z, L_{\mathrm{c}}\right),
\end{aligned}
$$

where, based on the radial symmetry of the system, we have considered $\mathrm{G}_{l x}^{\mathrm{o}}=\mathrm{G}_{l y}^{\mathrm{o}}$. For the $\mathrm{E}_{2 \mathrm{~g} 2}$ symmetry, the $\mathbf{S T}$ component assumes the form

$$
\begin{aligned}
& S^{\mathrm{ST}}\left(\mathbf{r}_{0}\right)\left(\mathrm{E}_{2 \mathrm{~g} 2}\right) \\
& =\frac{9}{64} \frac{\omega_{\mathrm{s}}^{4}}{\varepsilon_{0}^{4} c^{4}} \sum_{l}\left|\mathrm{G}_{l x}^{\mathrm{o}}\left(\mathbf{r}_{0}, z ; \omega_{\mathrm{s}}\right) \alpha_{\|}(\omega) E_{0 z}(z, \omega)\right|^{2} \\
& \quad \times\left(\tilde{\alpha}_{x y}^{\gamma *} \tilde{\alpha}_{x y}^{\gamma}+\tilde{\alpha}_{y x}^{\gamma *} \tilde{\alpha}_{y x}^{\gamma}\right) \ell_{x x}\left(z, L_{\mathrm{c}}\right) .
\end{aligned}
$$

Expressing $\alpha_{\|}$in terms of the complex field-enhancement factor $f_{\mathrm{e}}(\omega)$ and the tip radius $r_{\text {tip }}$ [Eq. (22)], and inserting the Raman tensor components [Eq. (9)] into Eqs. (49) and (50), we obtain the following expressions for the $\mathrm{G}$ and $\mathrm{G}^{\prime}$ Raman signals:

$$
\begin{aligned}
S_{\mathrm{G}}^{\mathrm{ST}}\left(\mathbf{r}_{0}\right)= & \frac{9}{16} \frac{\omega_{\mathrm{s}}^{4}}{\varepsilon_{0}^{2} c^{4}} r_{\text {tip }}^{6} \tilde{f}_{\mathrm{e}}(\omega)^{2} \sum_{l} \mid \mathrm{G}_{l x}^{\mathrm{o}}\left(\mathbf{r}_{0}, z ; \omega_{\mathrm{s}}\right) \\
& \times\left. E_{0 z}(z, \omega) \tilde{\alpha}^{\mathrm{G}}\left(\omega_{\mathrm{s}}, \omega\right)\right|^{2} \ell_{x x}\left(z, L_{\mathrm{c}}\right), \\
S_{\mathrm{G}^{\prime}}^{\mathrm{ST}}\left(\mathbf{r}_{0}\right)= & \frac{9}{32} \frac{\omega_{\mathrm{s}}^{4}}{\varepsilon_{0}^{2} c^{4}} r_{\text {tip }}^{6} \tilde{f}_{\mathrm{e}}(\omega)^{2} \sum_{l} \mid \mathrm{G}_{l x}^{\mathrm{o}}\left(\mathbf{r}_{0}, z ; \omega_{\mathrm{s}}\right) \\
& \times\left. E_{0 z}(z, \omega) \tilde{\alpha}^{\mathrm{G}^{\prime}}\left(\omega_{\mathrm{s}}, \omega\right)\right|^{2} \ell_{x x}\left(z, L_{\mathrm{c}}\right) .
\end{aligned}
$$

Equations (51) and (52) show that, unlike the TST case, the only differences between the $\mathrm{G}$ and $\mathrm{G}^{\prime} \mathbf{S T}$ signals are their numerical prefactors and Raman efficiencies, expressed in terms of $\tilde{\alpha}^{\mathrm{G}}$ and $\tilde{\alpha}^{\mathrm{G}^{\prime}}$. Figure 8 shows the plot of the distance dependence of the $G$ and $G^{\prime}$ ST signals [according to Eqs. (51) and (52), respectively] for different correlation lengths $L_{\mathrm{c}}$, assuming $r_{\text {tip }}=15 \mathrm{~nm}$ and $\tilde{f}_{\mathrm{e}}=3$. The signal is normalized to 1 at $z_{\min }=20 \mathrm{~nm}$. As expected, the $\mathrm{G}$ and $\mathrm{G}^{\prime}$ curves coincide for all values of $L_{\mathrm{c}}$. For $L_{\mathrm{c}}=0$, they show a dependence on $z^{-4}$. For a finite correlation length $L_{\mathrm{c}}$, we observe a slight drop in the $z$ dependence. For $L_{\mathrm{c}} \rightarrow \infty$, neither of them shows enhancement.

TST/ID.-Next, we evaluate the TST component of the near-field signal for 1D systems, more specifically, for the D band at graphene edges. The $\mathrm{D}$ mode is totally symmetric ( $\mathrm{A}_{1}$ symmetry), and we consider the edge along the $x$ direction, with coordinate $y=0$. In this case, the position vector at the sample is reduced to $\mathbf{r}=(x, 0,0)$. An important factor to be taken into account is the strong depolarization effect in the optical absorption of 1D systems, for which the absorption is maximum for light polarized along the longitudinal direction of the object and null for light 

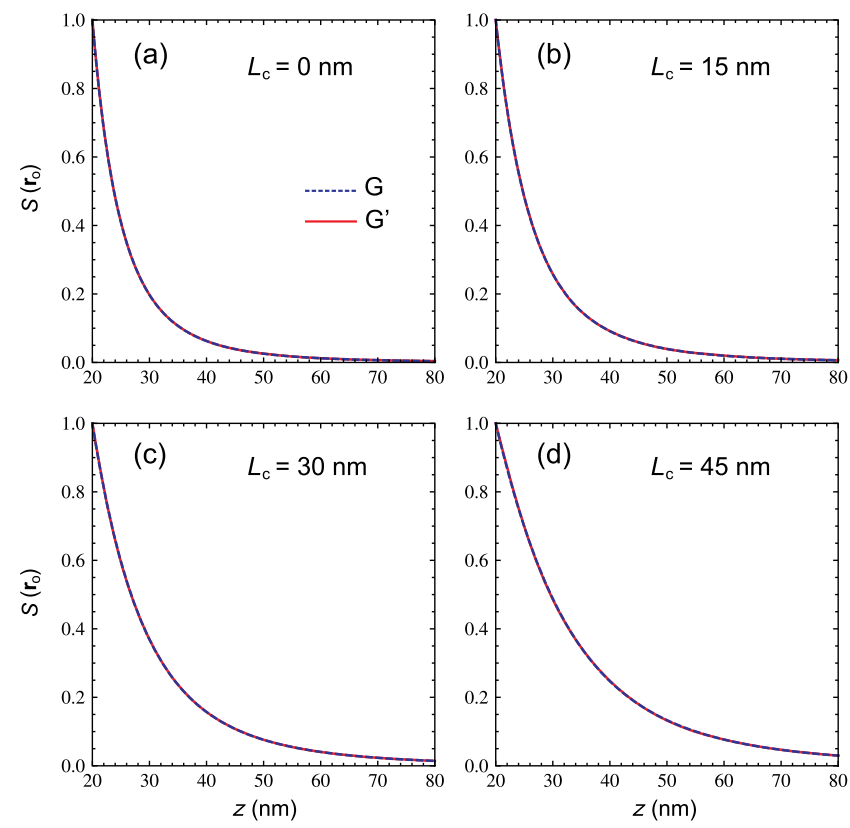

FIG. 8. Distance dependence of the ST signal for the $\mathrm{G}$ and $\mathrm{G}^{\prime}$ bands. Panels (a-d) account for different values of the correlation length $L_{\mathrm{c}}: 0,15,30$, and $45 \mathrm{~nm}$, respectively, as indicated in the graphics. In all cases, we used $\tilde{f}_{\mathrm{e}}=3$ and $r_{\text {tip }}=15 \mathrm{~nm}$. The signal $S\left(\mathbf{r}_{0}\right)$ is normalized to 1 at $z_{\min }=20 \mathrm{~nm}$. Compared to the TST signal shown in Fig. 5, the ST signal presents a weaker decay and therefore contributes to the measured signal only for larger tip-sample distances. polarized along its transverse direction $[20,30]$. To account for depolarization, we introduce a depolarization tensor $\stackrel{\leftrightarrow}{d}$ with which the local excitation field [Eq. (24)] becomes

$$
\begin{aligned}
\mathbf{E}_{\mathrm{tot}}(x, \omega) \\
\quad=\stackrel{\leftrightarrow}{d}\left[\mathbf{E}_{0}(x, \omega)+\frac{\omega^{2}}{\varepsilon_{0} c^{2}} \stackrel{\leftrightarrow}{\mathrm{G}^{\mathrm{o}}}(x, z ; \omega) \stackrel{\leftrightarrow}{\mathrm{\alpha}_{\mathrm{tip}}}(\omega) \mathbf{E}_{0}(z, \omega)\right],
\end{aligned}
$$

with

$$
\stackrel{\leftrightarrow}{d}(\omega)\left[\begin{array}{lll}
1 & 0 & 0 \\
0 & 0 & 0 \\
0 & 0 & 0
\end{array}\right]
$$

The Raman induced dipole [Eq. (4)] is now evaluated as

$$
\mathbf{p}^{\gamma}\left(x, \omega_{\mathrm{s}}\right)=\stackrel{\leftrightarrow}{\alpha}^{\gamma}\left(x, \omega_{\mathrm{s}} ; \omega\right) \mathbf{E}_{\mathrm{tot}}(x, \omega)
$$

The same depolarization effect accounts for the scattered field and, in this case, Eq. (3) becomes

$\mathbf{E}\left(\mathbf{r}_{0}, \omega_{s}\right)=\stackrel{\leftrightarrow}{d}\left[\frac{\omega_{\mathrm{s}}^{2}}{\varepsilon_{0} c^{2}} \int_{-\infty}^{+\infty} d x \stackrel{\leftrightarrow}{\mathrm{G}}\left(\mathbf{r}_{0}, x ; \omega_{\mathrm{s}}\right) \mathbf{p}^{\gamma}\left(x ; \omega_{\mathrm{s}}\right)\right]$

For totally symmetric modes in 1D systems, the scattered signal in the TST configuration [Eq. (16) for the 2D case] is given by

$$
S\left(\mathbf{r}_{0}\right)=2 \pi \frac{\omega_{\mathrm{s}}^{4}}{\varepsilon_{0}^{2} c^{4}} \sum_{l} \tilde{\alpha}_{x x}^{\gamma *} \tilde{\alpha}_{x x}^{\gamma} \int_{-\infty}^{+\infty} d k_{x} \hat{F}_{l x}^{*}\left(k_{x}\right) \hat{F}_{l x}\left(k_{x}\right) \mathrm{e}^{-\left(k_{x}^{2} L_{\mathrm{c}}^{2}\right) / 4}
$$

The Fourier components $\hat{F}_{l x}^{*}\left(k_{x}\right)$ in Eq. (57) can be evaluated as

$$
\hat{F}_{l x}\left(k_{x}\right)=\frac{1}{2 \pi} \frac{\omega^{2} \omega_{\mathrm{s}}^{2}}{\varepsilon_{0}^{2} c^{4}} \alpha_{\|}(\omega) \alpha_{\|}\left(\omega_{\mathrm{s}}\right) \mathrm{G}_{l z}^{\mathrm{o}}\left(\mathbf{r}_{0}, z ; \omega_{\mathrm{s}}\right) E_{0 z}(z, \omega) \times \int_{-\infty}^{+\infty} d x \mathrm{G}_{z x}^{\mathrm{o}}\left(x, y, z ; \omega_{\mathrm{s}}\right) \mathrm{G}_{x z}^{\mathrm{o}}(x, y, z ; \omega) \mathrm{e}^{-i k_{x} x} .
$$

Using the same approximations as for the TST scattering in 2D systems [see Eq. (31)], we obtain

$$
\hat{F}_{l x}\left(k_{x}\right)=\frac{9}{128 \pi^{5 / 2} \varepsilon_{0}^{2} z^{5}} \alpha_{\|}(\omega) \alpha_{\|}\left(\omega_{\mathrm{s}}\right) \mathrm{G}_{l z}^{\mathrm{o}}\left(\mathbf{r}_{0}, z ; \omega_{\mathrm{s}}\right) E_{0 z}(z, \omega) \hat{h}_{x}\left(k_{x} ; z\right),
$$

where

$$
\hat{h}_{x}\left(k_{x} ; z\right)=\left[\frac{a_{0}\left(2 b_{0}-k_{x}^{2} z^{2}\right)}{b_{0}^{5 / 2}} \mathrm{e}^{-\left(k_{x}^{2} z^{2}\right) / 4 b_{0}}+\frac{c_{0}\left(2 d_{0}-k_{x}^{2} z^{2}\right)}{d_{0}^{5 / 2}} \mathrm{e}^{-\left(k_{x}^{2} z^{2}\right) / 4 d_{0}}\right],
$$

with $a_{0}=0.74, b_{0}=4.0, c_{0}=0.08$, and $d_{0}=1.5$ (same values as those obtained for the TST scattering in 2D systems). To calculate the Raman signal (57), we define the expression

$$
\ell_{x x}\left(z, L_{\mathrm{c}}\right)=\int_{-\infty}^{+\infty} d k_{x} \hat{h}_{x}^{*}\left(k_{x} ; z\right) \hat{h}_{x}\left(k_{x} ; z\right) \mathrm{e}^{-\left(k_{x}^{2} L_{\mathrm{c}}^{2}\right) / 4},
$$

which can be solved analytically. It has the properties 


$$
\ell_{x x}\left(z, L_{\mathrm{c}} \rightarrow \infty\right)=1 / L_{\mathrm{c}}, \quad \ell_{x x}\left(z, L_{\mathrm{c}} \rightarrow 0\right)=1 / z .
$$

Inserting Eq. (61) into Eq. (57) yields

$$
S^{\mathrm{TST}}\left(\mathbf{r}_{0}\right)=\frac{81}{512} \frac{\omega_{\mathrm{s}}^{4}}{\varepsilon_{0}^{2} c^{4}} \frac{r_{\mathrm{tip}}^{12} \tilde{f}_{\mathrm{e}}(\omega)^{4}}{z^{10}} \sum_{l}\left|\mathrm{G}_{l z}^{\mathrm{o}}\left(\mathbf{r}_{0}, z ; \omega_{\mathrm{s}}\right) E_{0 z}(z, \omega) \tilde{\alpha}^{\mathrm{D}}\left(\omega_{\mathrm{s}} ; \omega\right)\right|^{2} \ell_{x x}\left(z, L_{\mathrm{c}}\right),
$$

where we have used $\tilde{\alpha}_{x x}^{\gamma}=1$, according to Eq. (9). We find that for a 1D system with zero correlation length $\left(L_{\mathrm{c}} \rightarrow 0\right)$, the TST signal from a totally symmetric mode decays as $z^{-11}$, whereas for infinite correlation length $\left(L_{\mathrm{c}} \rightarrow \infty\right)$, it decays as $z^{-10}$, consistent with the theory described in Ref. [25]. Figure 9 shows the plot of the distance dependence of the TST signal for the D band [according to Eq. (63)] for different correlation lengths $L_{\mathrm{c}}$, assuming $r_{\text {tip }}=15 \mathrm{~nm}$ and $\tilde{f}_{\mathrm{e}}=3$. The signal is normalized to 1 at $z_{\min }=20 \mathrm{~nm}$. The smaller the correlation length, the steeper the distance dependence.

ST/1D.-Starting from Eq. (16), the ST component of the scattered signal for the $1 \mathrm{D}$ case can be calculated as

$$
\begin{aligned}
S^{\mathrm{ST}}\left(\mathbf{r}_{0}\right)= & 2 \pi \frac{\omega_{\mathrm{s}}^{4}}{\varepsilon_{0}^{2} c^{4}} \sum_{l} \tilde{\alpha}_{x x}^{\gamma *} \tilde{\alpha}_{x x}^{\gamma} \\
& \times \int_{-\infty}^{+\infty} d k_{x} ; \hat{F}_{l x}^{*}\left(k_{x}\right) \hat{F}_{l x}\left(k_{x}\right) \mathrm{e}^{-\left(k_{x}^{2} L_{\mathrm{c}}^{2}\right) / 4},
\end{aligned}
$$

where, according to Eq. (26), the Fourier $\hat{F}_{l x}\left(k_{x}\right)$ component has the form
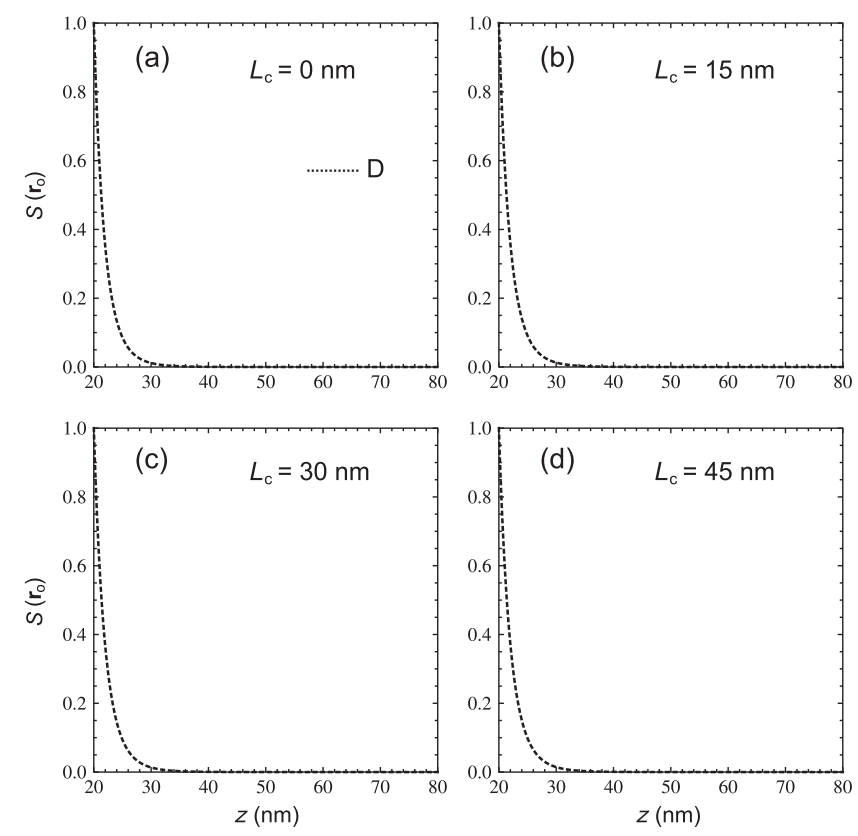

FIG. 9. Dependence of the TST of the D band on tip-sample separation $z$. Panels (a-d) account for different values of $L_{\mathrm{c}}: 0,15$, 30 , and $45 \mathrm{~nm}$, respectively, as indicated in the graphics. In all cases, we used $\tilde{f}_{\mathrm{e}}=3$ and $r_{\text {tip }}=15 \mathrm{~nm}$. The signal $S\left(\mathbf{r}_{0}\right)$ is normalized to 1 at $z_{\min }=20 \mathrm{~nm}$.

$$
\begin{aligned}
\hat{F}_{l x}\left(k_{x}\right)= & \frac{1}{2 \pi} \frac{\omega^{2}}{\varepsilon_{0} c^{2}} \alpha_{\|}(\omega) \mathrm{G}_{l x}^{\mathrm{o}}\left(\mathbf{r}_{0}, z ; \omega_{\mathrm{s}}\right) E_{0 z}(z, \omega) \\
& \times \int_{-\infty}^{+\infty} d x \mathrm{G}_{x z}^{\mathrm{o}}(x, z ; \omega) \mathrm{e}^{-i k_{x} x} .
\end{aligned}
$$

Considering the same approximations as for the 2D case [Eq. (43)], the Fourier component (65) can be evaluated as

$$
\hat{F}_{l x}\left(k_{x}\right)=\frac{(-i) 3}{16 \pi^{3 / 2} \varepsilon_{0} z} \alpha_{\perp}(\omega) \mathrm{G}_{l x}^{\mathrm{o}}\left(\mathbf{r}_{0}, z ; \omega_{\mathrm{s}}\right) E_{0 z}(z, \omega) \hat{h}_{x}\left(k_{x}\right),
$$

with

$$
\hat{h}_{x}\left(k_{x}\right)=k_{x}\left[\frac{a_{0}^{\prime}}{b_{0}^{\prime 3 / 2}} \mathrm{e}^{-\left(k_{x} z\right)^{2} / 4 b_{0}^{\prime}}+\frac{c_{0}^{\prime}}{d_{0}^{13 / 2}} \mathrm{e}^{-\left(k_{x} z\right)^{2} / 4 d_{0}^{\prime}}\right],
$$

where the fitting parameters $a_{0}^{\prime}, b_{0}^{\prime}, c_{0}^{\prime}$, and $d_{0}^{\prime}$ are the same as those obtained for the $2 \mathrm{D}$ case $\left(a_{0}^{\prime}=0.78, b_{0}^{\prime}=2.4\right.$, $c_{0}^{\prime}=0.18$, and $\left.d_{0}^{\prime}=0.56\right)$. We introduce the function

$$
\ell_{x x}\left(z, L_{\mathrm{c}}\right)=\int_{-\infty}^{+\infty} d k_{x} \hat{h}_{x}^{*}\left(k_{x}\right) \hat{h}_{x}\left(k_{x}\right) \mathrm{e}^{-\left(k_{x}^{2} L_{\mathrm{c}}^{2}\right) / 4},
$$

which can be calculated analytically and has the properties $\ell_{x x}\left(z, L_{\mathrm{c}} \rightarrow \infty\right)=1 / L_{\mathrm{c}}^{3}, \quad \ell_{x x}\left(z, L_{\mathrm{c}} \rightarrow 0\right)=1 / z^{3}$.

Inserting Eqs. (66)-(68) into Eq. (64) yields

$$
\begin{aligned}
S^{\mathrm{ST}}\left(\mathbf{r}_{0}\right)= & \frac{9}{32} \frac{\omega_{\mathrm{s}}^{4}}{\varepsilon_{0}^{2} c^{4} z^{2}} r_{\mathrm{tip}}^{6} \tilde{f}_{\mathrm{e}}(\omega)^{2} \\
& \times \sum_{l}\left|\mathrm{G}_{l x}^{\mathrm{o}}\left(\mathbf{r}_{0}, z ; \omega_{\mathrm{s}}\right) E_{0 z}(z, \omega) \tilde{\alpha}_{0}^{\mathrm{G}}\left(\omega_{\mathrm{s}}, \omega\right)\right|^{2} \ell_{x x}\left(z, L_{\mathrm{c}}\right),
\end{aligned}
$$

where we have used $\tilde{\alpha}_{x x}^{\gamma}=1$. According to Eqs. (69) and (70), for $L_{\mathrm{c}}=0$, the $\mathrm{D}$ band signal in the $\mathbf{S T}$ configuration is proportional to $z^{-5}$, and for $L_{\mathrm{c}} \rightarrow \infty$, the signal is proportional to $z^{-2}$ (see Fig. 10). Similar to the $2 \mathrm{D}$ case for the Raman $G$ and $G^{\prime}$ bands, we find that the ST term has a weaker distance dependence than the TST term, and therefore, it contributes to the measured signal only for large tipsample distances. It should be noticed that, unlike the $2 \mathrm{D}$ case for which the lateral position of the tip is irrelevant, for 

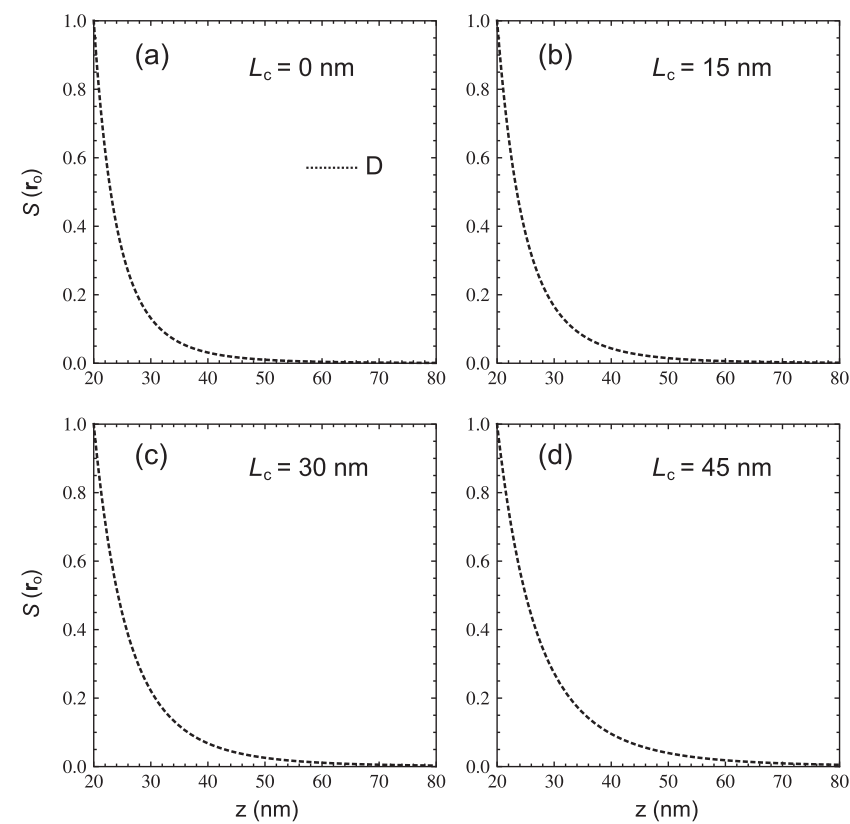

FIG. 10. Distance dependence of the ST signal for the D band. Panels (a-d) account for different values of $L_{\mathrm{c}}: 0,15,30$, and $45 \mathrm{~nm}$, respectively, as indicated in the graphics. In all cases, we set $\tilde{f}_{\mathrm{e}}=3$ and $r_{\text {tip }}=15 \mathrm{~nm}$. The signal $S\left(\mathbf{r}_{0}\right)$ is normalized to 1 at $z_{\min }=20 \mathrm{~nm}$.

1D systems, the symmetry is broken at $y=0$ (considering the sample along the $x$ direction). Therefore, the correlation length $L_{\mathrm{c}}$ could also be determined in $1 \mathrm{D}$ systems by varying the position of the tip along the $y$ direction.

Finally, we summarize our findings and discuss the main results. We have presented the theory of near-field Raman scattering accounting for spatial source correlations and associated coherence properties of the scattered signal. Our calculations were performed for a TERS configuration, where a metal tip acting as an optical antenna is positioned near the sample. We considered the TST and ST components of the scattered field, and the calculations were performed for $1 \mathrm{D}$ and $2 \mathrm{D}$ scatterers. The theory was applied specifically to graphene, namely, the D, G, and $\mathrm{G}^{\prime}$ Raman bands. While the $\mathrm{D}$ and $\mathrm{G}^{\prime}$ bands are associated with totally symmetric $\left(A_{1}\right)$ phonons, the $G$ band originates from a double degenerate mode with $\mathrm{E}_{2 \mathrm{~g}}$ symmetry. On the other hand, while the $\mathrm{G}$ and $\mathrm{G}^{\prime}$ bands are allowed over the whole graphene area, the $\mathrm{D}$ band is strongly localized at the edges which define a one-dimensional scatterer.

For samples with finite correlation length $L_{\mathrm{c}}$, the TST term gives rise to a characteristic difference between the $\mathrm{G}$ and $\mathrm{G}^{\prime}$ Raman signals. This difference is associated with near-field interferences which, in the case of the Raman $\mathrm{G}$ band, are destructive, and in the case of the $\mathrm{G}^{\prime}$ band, turn out to be constructive (see Fig. 6 and associated discussion). The near-field interferences give rise to a weaker tip-sample distance dependence for the $\mathrm{G}$ band than for the $\mathrm{G}^{\prime}$ band, and they make it possible to extract the correlation length $L_{\mathrm{c}}$
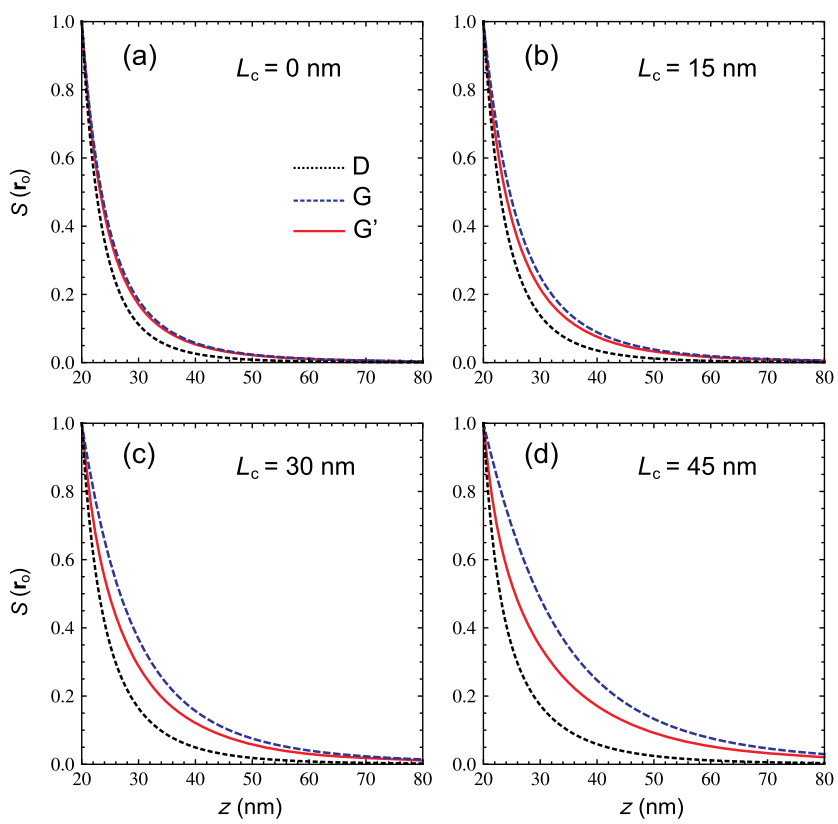

FIG. 11. Distance dependence of the TST $+\mathbf{S T}$ signals for the $\mathrm{D}, \mathrm{G}$, and $\mathrm{G}^{\prime}$ bands. Panels (a-d) account for different values of $L_{\mathrm{c}}: 0,15,30$, and $45 \mathrm{~nm}$, respectively, as indicated in the graphics. In all cases, we used $f_{\mathrm{e}}=3$ and $r_{\text {tip }}=15 \mathrm{~nm}$. The signal $S\left(\mathbf{r}_{0}\right)$ is normalized to 1 at $z_{\min }=20 \mathrm{~nm}$.

from measured data. Moreover, when the D band signal originates from the edges (1D geometry), a further modification of the distance dependence is observed. All these effects are summarized in Fig. 11, which shows the plot of the TST + ST signal versus the tip-sample separation $(z)$ for different values of $L_{\mathrm{c}}(0,15,30$, and $45 \mathrm{~nm})$. In all cases, we used $\tilde{f}_{\mathrm{e}}=3$ and $r_{\text {tip }}=15 \mathrm{~nm}$. The $\mathrm{G}$ and $\mathrm{G}^{\prime}$ curves coincide for $L_{\mathrm{c}}=0$, as expected. The $\mathrm{D}$ band signal presents a different trend, showing a steeper tip-sample distance dependence (stronger enhancement). For finite values of $L_{\mathrm{c}}$, the distance dependence drops for all bands as $L_{\mathrm{c}}$ increases. Simultaneously, $\mathrm{G}$ and $\mathrm{G}^{\prime}$ distance curves become different, with the $\mathrm{G}$ band showing a markedly weaker enhancement. Therefore, the experimental observation of different tip-sample distance dependencies for the $\mathrm{G}$ and $\mathrm{G}^{\prime}$ bands provides strong evidence for near-field interference effects associated with finite correlation lengths $L_{\mathrm{c}}$.

The curves shown in Fig. 11 were reproduced in experimental measurements in Ref. [15], where we have measured the near-field Raman signal of the D, G, and $\mathrm{G}^{\prime}$ bands on graphene samples. In these experiments, the correlation length of the $\mathrm{G}$ and $\mathrm{D}$ optical phonons were obtained by fitting the near-field experimental data with the theory presented here. For both cases ( $D$ and $G$ phonons), we found $L_{\mathrm{c}} \approx 30 \mathrm{~nm}$. Note that it is possible to extract $L_{\mathrm{c}}$ from the width of the Raman lines in nano-structured systems [31], but the obtained value is an average over the laser-irradiated sample area. On the other hand, the near-field procedure developed here allows for the measurement of $L_{\mathrm{c}}$ in single 
crystals and with nanoscale spatial resolutions, which makes it applicable to the analysis of transport properties of a wide range of materials. Most importantly, our work demonstrates that it is not a priori legitimate to treat Raman scattering as an incoherent process in which the signal from different sample regions is simply summed up.

We are grateful for valuable input from P. Scott Carney. R. B. and L. N. acknowledge financial support from the U.S. Department of Energy (Grant No. DE-FG0205ER46207) and the Swiss National Science Foundation (Grant No. 200021_149433). L. G. C. and A. J. acknowledge financial support from CNPq and FAPEMIG. A. J. acknowledges CAPES for financing his stay at ETH.

[1] M. Cardona and G. Guntherodt, Resonance Phenomena, Light Scattering in Solids II: Basic Concepts and Instrumentation, Topics in Applied Physics Vol. 50 (Springer-Verlag, Berlin, 1982), pp. 19-98.

[2] W. Hayes and R. Loudon, Scattering of Light by Crystals (John Wiley \& Sons, New York, 1978).

[3] W. H. Carter and E. Wolf, Coherence Properties of Lambertian and Non-Lambertian Sources, J. Opt. Soc. Am. 65, 1067 (1975).

[4] R. Carminati and J.-J. Greffet, Near-Field Effects in Spatial Coherence of Thermal Sources, Phys. Rev. Lett. 82, 1660 (1999).

[5] A. V. Shchegrov, K. Joulain, R. Carminati, and J.-J. Greffet, Near-Field Spectral Effects Due to Electromagnetic Surface Excitations, Phys. Rev. Lett. 85, 1548 (2000).

[6] H. Roychowdhury and E. Wolf, Effects of Spatial Coherence on Near-Field Spectra, Opt. Lett. 28, 170 (2003).

[7] A. Apostol and A. Dogariu, Spatial Correlations in the Near Field of Random Media, Phys. Rev. Lett. 91, 093901 (2003).

[8] H. Roychowdhury and E. Wolf, Coherence Effects in the Near-Field, J. Mod. Opt. 51, 1603 (2004).

[9] K. Kang, D. Abdula, D. G. Cahill, and M. Shim, Lifetimes of Optical Phonons in Graphene and Graphite by TimeResolved Incoherent Anti-Stokes Raman Scattering, Phys. Rev. B 81, 165405 (2010).

[10] S. Berciaud, M. Y. Han, K. F. Mak, L. E. Brus, P. Kim, and T. F. Heinz, Electron and Optical Phonon Temperatures in Electrically Biased Graphene, Phys. Rev. Lett. 104, 227401 (2010).

[11] H. Wang, J. H. Strait, P. A. George, S. Shivaraman, V. B. Shields, M. Chandrashekhar, J. Hwang, F. Rana, M. G. Spencer, C. S. Ruiz-Vargas, and J. Park, Ultrafast Relaxation Dynamics of Hot Optical Phonons in Graphene, Appl. Phys. Lett. 96, 081917 (2010).

[12] S. Wu, W.-T. Liu, X. Liang, P. J. Schuck, F. Wang, Y. R. Shen, and M. Salmeron, Hot Phonon Dynamics in Graphene, Nano Lett. 12, 5495 (2012).

[13] B. Y. Sun, Y. Zhou, and M. W. Wu, Dynamics of Photoexcited Carriers in Graphene, Phys. Rev. B 85, 125413 (2012).
[14] G. D. Sanders, A. R. T. Nugraha, K. Sato, J.-H. Kim, J. Kono, R. Saito, and C. J. Stanton, Theory of Coherent Phonons in Carbon Nanotubes and Graphene Nanoribbons, J. Phys. Condens. Matter 25, 144201 (2013).

[15] R. Beams, L. G. Cançado, A. Jorio, and L. Novotny, Spatial Coherence in Raman Scattering (unpublished).

[16] T. Dekorsy, G. C. Cho, and H. Kurz, Coherent Phonons in Condensed Media, Scattering in Solids VIII, Topics in Applied Physics Vol. 76 (Springer, Berlin, 2000), pp. 169-209.

[17] E. Wolf, Introduction to the Theory of Coherence and Polarization of Light (Cambridge University Press, New York, 2007).

[18] A. Jorio, M. S. Dresselhaus, R. Saito, and G. Dresselhaus, Raman Spectroscopy in Graphene Related Systems (Wiley-VCH, Weinheim, 2011).

[19] A. C. Ferrari and D. M. Basko, Raman Spectroscopy as a Versatile Tool for Studying the Properties of Graphene, Nat. Nanotechnol. 8, 235 (2013).

[20] C. Casiraghi, A. Hartschuh, H. Qian, S. Piscanec, C. Georgi, A. Fasoli, K. S. Novoselov, D. M. Basko, and A. C. Ferrari, Raman Spectroscopy of Graphene Edges, Nano Lett. 9, 1433 (2009).

[21] M. M. Lucchese, F. Stavale, E. H. Ferriera, C. Vilane, M. V. O. Moutinho, R. B. Capaz, C. A. Achete, and A. Jorio, Quantifying Ion-Induced Defects and Raman Relaxation Length in Graphene, Carbon 48, 1592 (2010).

[22] R. Beams, L. G. Cançado, and L. Novotny, LowTemperature Raman Study of the Electron Coherence Length Near Graphene Edges, Nano Lett. 11, 1177 (2011).

[23] W. Su and D. Roy, Visualizing Graphene Edges Using TipEnhanced Raman Spectroscopy, J. Vac. Sci. Technol. B 31, 041808 (2013).

[24] R. Loudon, The Raman Effect in Crystals, Adv. Phys. 13, 423 (1964).

[25] L. G. Cançado, A. Jorio, A. Ismach, E. Joselevich, A. Hartschuh, and L. Novotny, Mechanism of Near-Field Raman Enhancement in One-Dimensional Systems, Phys. Rev. Lett. 103, 186101 (2009).

[26] R. V. Maximiano, R. Beams, L. Novotny, A. Jorio, and L. G. Cançado, Mechanism of Near-Field Raman Enhancement in Two-Dimensional Systems, Phys. Rev. B 85, 235434 (2012).

[27] L. Novotny and B. Hecht, Principles of Nano-Optics (Cambridge University Press, New York, 2012).

[28] J. Sun, J. C. Schotland, and P. S. Carney, Strong Probe Effects in Near-Field Optics, J. Appl. Phys. 102, 103103 (2007).

[29] A. Hartschuh, E. J. Sánchez, X. S. Xie, and L. Novotny, High-Resolution Near-Field Raman Microscopy of SingleWalled Carbon Nanotubes, Phys. Rev. Lett. 90, 095503 (2003).

[30] L. G. Cançado, M. A. Pimenta, B. R. A. Neves, M. S. S. Dantas, and A. Jorio, Influence of the Atomic Structure on the Raman Spectra of Graphite Edges, Phys. Rev. Lett. 93, 247401 (2004).

[31] H. Richter, Z. P. Wang, and L. Ley, The One Phonon Raman Spectrum in Microcrystalline Silicon, Solid State Commun. 39, 625 (1981). 